\title{
ROTATION IN THE PLEIADES WITH K2. III. SPECULATIONS ON ORIGINS AND EVOLUTION
}

\author{
John Stauffer $^{1}$, Luisa Rebull ${ }^{1}$, Jerome Bouvier ${ }^{2,3}$, Lynne A. Hillenbrand ${ }^{4}$, Andrew Collier-Cameron ${ }^{5}$, \\ Marc Pinsonneault ${ }^{6}$, Suzanne Aigrain ${ }^{7}$, David Barrado ${ }^{8}$, Herve Bour $^{8}$, David Ciardi $^{9}$, Ann Marie Cody $^{10}$,

\begin{abstract}
We use high-quality $K 2$ light curves for hundreds of stars in the Pleiades to better understand the angular momentum evolution and magnetic dynamos of young low-mass stars. The $K 2$ light curves provide not only rotational periods but also detailed information from the shape of the phased light curve that was not available in previous studies. A slowly rotating sequence begins at $\left(V-K_{\mathrm{s}}\right)_{0} \sim 1.1$ (spectral type F5) and ends at $\left(V-K_{\mathrm{s}}\right)_{0} \sim 3.7$ (spectral type K8), with periods rising from $\sim 2$ to $\sim 11$ days in that interval. A total of $52 \%$ of the Pleiades members in that color interval have periods within $30 \%$ of a curve defining the slow sequence; the slowly rotating fraction decreases significantly redward of $\left(V-K_{\mathrm{s}}\right)_{0}=2.6$. Nearly all of the slow-sequence stars show light curves that evolve significantly on timescales less than the $K 2$ campaign duration. The majority of the FGK Pleiades members identified as photometric binaries are relatively rapidly rotating, perhaps because binarity inhibits star-disk angular momentum loss mechanisms during pre-main-sequence evolution. The fully convective late $\mathrm{M}$ dwarf Pleiades members $\left(5.0<\left(V-K_{\mathrm{s}}\right)_{0}<6.0\right)$ nearly always show stable light curves, with little spot evolution or evidence of differential rotation. During pre-main-sequence evolution from 3 Myr (NGC 2264 age) to $\sim 125 \mathrm{Myr}$ (Pleiades age), stars of $0.3 M_{\odot}$ shed about half of their angular momentum, with the fractional change in period between 3 and 125 Myr being nearly independent of mass for fully convective stars. Our data also suggest that very low mass binaries form with rotation periods more similar to each other and faster than would be true if drawn at random from the parent population of single stars.
\end{abstract}

Key words: open clusters and associations: individual (Pleiades) - stars: rotation

Supporting material: machine-readable table

\section{INTRODUCTION}

The Kepler main mission produced multiyear light curves for $>100,000$ low-mass stars with exquisite precision and unprecedented cadence and duration. Those light curves were primarily designed to identify exoplanet transits and to determine the frequency of terrestrial planets around low-mass host stars. The mission was remarkably successful, identifying hundreds of now-confirmed exoplanets (Batalha 2014) and thousands of additional exoplanet candidates (Rowe et al. 2015).

In addition to the main exoplanet science, the high quality and long duration of the Kepler light curves enabled many other types of science, including (among others) astroseismology (e.g., Aguirre et al. 2014), the identification and characterization of eclipsing binary stars (e.g., Prsa et al. 2011), rotation periods for low-mass field stars (e.g., McQuillan et al. 2014), the frequency of superflares in solar-mass stars (Maehara et al. 2012), the identification of long-lived clouds in the atmosphere of an $\mathrm{L}$ dwarf (Gizis et al. 2015), quantifying how clouds and latitudinal differential rotation affect the optical light curves of ice-giant planets (Simon et al. 2016), and active galactic nucleus reverberation mapping (e.g., Pei et al. 2014). However, the fixed position in the sky of the original Kepler field meant that a number of science topics amenable to study with high-quality light curves were only minimally addressed, or not at all.

K2, the extended Kepler mission (Howell et al. 2014), represents an unexpected opportunity to target additional locations in the Galaxy for specific science goals. One particular science topic that greatly benefits from the new $K 2$ mission is the study of rotation and rotation-related phenomena in young, low-mass stars. The original Kepler field contained no star-forming regions and no young (age $\leqslant 1 \mathrm{Gyr}$ ) open clusters. The $K 2$ fields were specifically designed to provide light curves for a sample of nearby, young pre-main-sequence (PMS) stars (Upper Sco and Taurus) and light curves for stars in several nearby, young open clusters (Pleiades, M35, Hyades, 
Praesepe). These light curves are providing a wealth of new data important for understanding the formation and evolution of low-mass stars, and in particular their angular momentum evolution.

The angular momentum evolution of young, low-mass stars has been a topic of great interest to stellar astronomers since at least the 1960s, when Bob Kraft published his studies of the spectroscopic rotational velocities $(v \sin i)$ of $\mathrm{A}, \mathrm{F}$, and early $\mathrm{G}$ dwarfs in the Alpha Per, Pleiades, Hyades, and Praesepe open clusters (Kraft 1965, 1966, 1967a, 1967b). Kraft (1970) showed that these data required that low-mass stars lose angular momentum over time, presumably due to stellar winds (Schatzmann 1962; Weber \& Davis 1967). Skumanich (1972) quantified this spin-down by showing that Kraft's data could be approximately fit by a $v_{\text {rot }} \propto t^{-1 / 2}$ relation. With improved detectors, later spectroscopic studies of many of the same open clusters were able to derive $v \sin i$ down into the $\mathrm{M}$ dwarf range (Stauffer \& Hartmann 1987; Stauffer et al. 1987; Soderblom et al. 1993a; Terndrup et al. 2000); these new data highlighted that for $M<1 M_{\odot}$ there is in fact a wide range in rotational velocities on the zero-age main sequence (ZAMS). This wide range in rotation decreases with time, such that by $\sim 600 \mathrm{Myr}$ all $\mathrm{G}$ dwarfs have very similar rotational velocities (Radick et al. 1987); the time for the rotational velocities to converge increases with decreasing mass (Stauffer et al. 1987). More recently, wide-field cameras on small telescopes with large fields of view have made it possible to obtain rotation periods for large samples of low-mass stars in many open clusters and star-forming regions, improving further the empirical database describing how low-mass stars shed their angular momentum over time. These data have motivated a large body of theoretical models that attempt with reasonable success to fit the distribution of rotation as a function of mass and age. Recent review articles that summarize the available rotational data and the theoretical models include Herbst et al. (2007, p. 297) and Bouvier et al. (2014, p. 433); see also Johnstone et al. (2015) for a thorough discussion of the spin-down models and their uncertainties.

Another key ingredient for understanding the angular momentum evolution of low-mass stars is their magnetic field topologies. Essentially all the angular momentum loss mechanisms depend on the surface magnetic fields and how those fields interact with the stellar wind or (during early PMS evolution) the star's circumstellar disk. It is only recently that detailed magnetic field topologies for low-mass stars have begun to become available, primarily from Zeeman Doppler imaging (ZDI) studies (e.g., Donati et al. 2008; Morin et al. 2008; Gregory et al. 2012). Because such studies can only be done with large telescopes and require considerable investment in telescope time, the number of stars that have been mapped is still small and the dependence of field topology on mass, age, and rotation rate is only beginning to be explored. Recent reviews of the available empirical data and inferences on how magnetic field topologies depend on mass and age can be found in Donati \& Landstreet (2009) and Linsky \& Scholler (2015).

The location of Field 4 on the $K 2$ mission was chosen in order to provide light-curve data for members of the Pleiades open cluster. Because of its proximity (136.2 pc; Melis et al. 2014), richness (more than 1500 probable members; Sarro et al. 2014), age (125 Myr; Stauffer et al. 1998), and the large body of published ancillary data, the Pleiades offers a strikingly good match to the capabilities of the $K 2$ mission. Within the $K 2$ field of view, $\sim 1000$ probable and possible Pleiades members could be targeted, ranging in mass from $>4 M_{\odot}$ to $<0.1 M_{\odot}$. In two companion papers, we describe the initial results of our analysis of the $K 2$ data. Rebull et al. (2016a, hereafter Paper I) determine periods for the Pleiades members with $K 2$ light curves and document the available broadband photometry for these stars. Rebull et al. (2016b, hereafter Paper II) identify stars with multiple periodic signals and provide extensive illustration of their light-curve signatures and interpretation. In this paper, we examine the distribution of rotation periods as a function of color (or, equivalently, mass) in the Pleiades and attempt to understand better why that distribution has its observed form. In Section 2, we briefly review the data we have available and the conclusions reached in Papers I and II. In Section 3, we discuss the slowly rotating main sequence in the Pleiades and possible mechanisms that may contribute to stars falling on that sequence and its shape at Pleiades age. We discuss the rotational velocities of Pleiades $M$ dwarfs in Section 4 and two sets of abnormally slowly rotating stars in Section 5 .

\section{OVERVIEW OF THE $K 2$ PLEIADES ROTATION DATA}

In order to set the stage for the remainder of the paper, in this section we briefly review the main points from Papers I and II; all of the detailed efforts to derive periods and light-curve shapes and for assembling the ancillary data needed to interpret the rotational data are provided in those papers. Reviews of the previous literature regarding rotation periods in the Pleiades and a table with cross-IDs between the $K 2$ EPIC identification numbers and the names by which the Pleiades members are known in the published literature are also provided in Paper I.

Light curves for of order 1000 candidate Pleiades members were obtained during the $K 2$ Field 4 campaign. This represents more than half of the known or suspected members of the cluster; most of the members lacking $K 2$ light curves simply fell outside the $K 2$ field of view (see Figure 1 of Paper I), and therefore we believe that the sample of cluster members with light curves should be relatively unbiased. As with any open cluster, the membership list includes stars with a wide range of pedigrees, ranging from essentially certain members to stars with only one study that suggested membership. We reassessed the membership of all the candidate stars with $K 2$ data, as described in Paper I, and have identified 826 stars we consider to be both good-quality members and in the brightness range where our extracted $K 2$ light curves are normally reliable. Of those, 759 have one or more significant periods from our periodogram analysis, and it is these 759 stars that are the topic of this paper.

When analyzing the rotation data, it is also necessary to have some proxy for the star's mass. We have $\left(V-I_{\mathrm{c}}\right)_{0}$ photometry for a large fraction of the FGK Pleiades members and will sometimes uses $\left(V-I_{\mathrm{c}}\right)_{0}$ as the proxy for mass in this spectral type range because the VI photometry is quite homogenous and the two bands were observed nearly simultaneously, mitigating the effect of variability on the derived color. However, we have chosen primarily to use $\left(V-K_{\mathrm{s}}\right)_{0}$ color as the mass proxy. Essentially all of the Pleiades members have Two Micron All Sky Survey (2MASS) $J H K_{\mathrm{s}}$ photometry (the 2MASS $10 \sigma$ limit of $K_{\mathrm{s}}=14.5$ corresponds to M6 spectral type in the Pleiades); many-but far from all-also have accurate, measured $V$ magnitudes. Where $V$ magnitudes are not known, we have used 


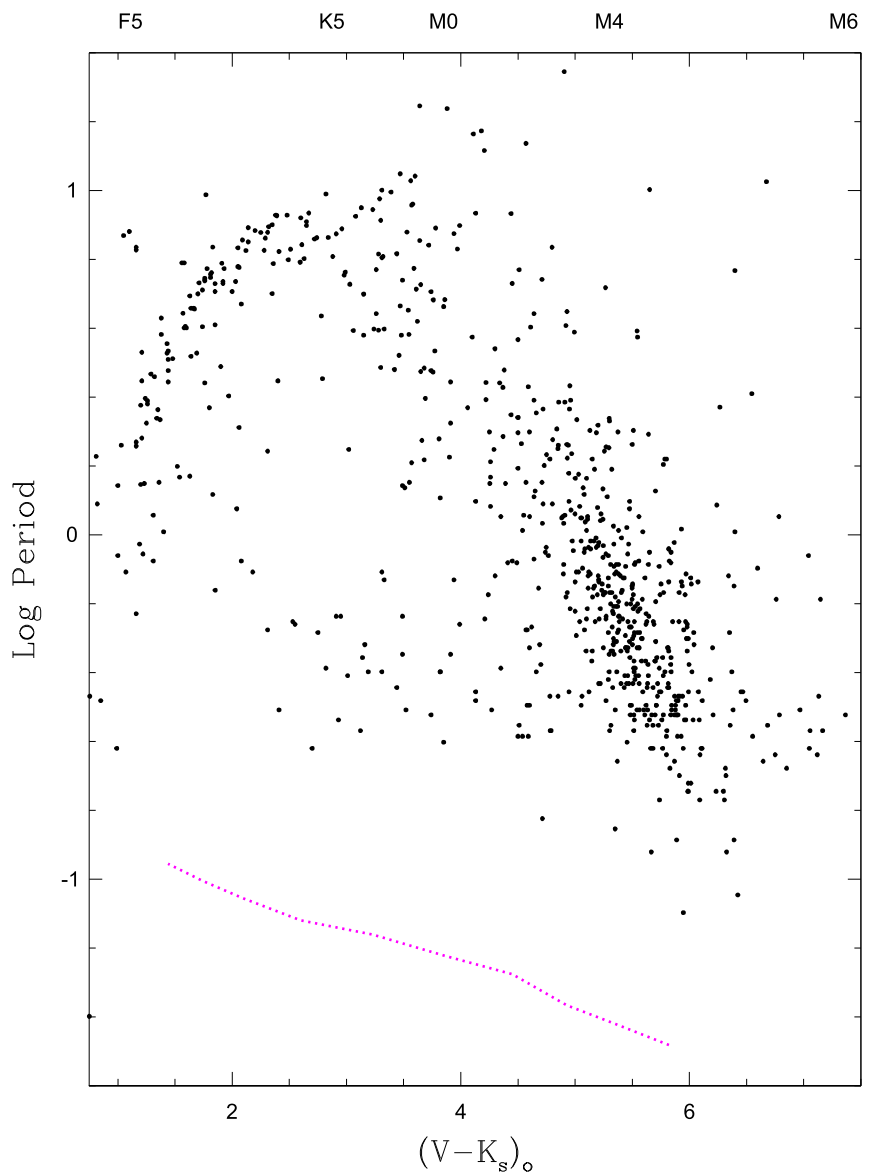

Figure 1. $K 2$ rotation periods for probable members of the Pleiades vs. their estimated $\left(V-K_{\mathrm{s}}\right)_{0}$ colors. The magenta curve shows an estimate of the breakup rotation period; even the most rapidly rotating low-mass Pleiades members are more than a factor of two away from the breakup period.

photometry at other wavelengths to estimate $\left(V-K_{\mathrm{s}}\right)_{0}$ colors (see Section 2.4 of Paper I). Figure 1 shows the main result from Paper $\mathrm{I}$ - the distribution of rotation periods for Pleiades members as a function of their $\left(V-K_{\mathrm{s}}\right)_{0}$ color.

The high quality of the $K 2$ data makes evident that a significant fraction (roughly a quarter) of the Pleiades members exhibit more than one statistically significant period in their light curves. Paper II discusses these multiply periodic systems in great detail and divides them into several categories: (a) pulsational variables, most notably $\delta$ Scuti stars (spectral type late A or early F; multiperiodic; periods typically $<0.1$ days; normally ascribed to $p$-mode pulsation); (b) stars with two or more very different periods, probably resulting from the target object being a binary, and each period being associated with one component of the binary; (c) stars with two or more relatively similar periods, either also ascribable to a binary system or possibly resulting from latitudinal differential rotation, spot evolution, and/or spot migration; and (d) stars showing phased light curves with two or more peaks or two or more minima, best explained as arising from spot groups centered at different longitudes. It is important to note that in Paper II, we have generally chosen to utilize nomenclature for the light-curve groups that are closely linked to the empirical shapes of the light curves and the periodogram features. In the discussion there, we propose physical processes that are the most likely mechanisms for generating these light-curve structures, but the primary terminology always emphasizes empirical features. Here, in Paper III, where the goals are to search for clues to physical processes, we often have chosen to utilize nomenclature that emphasizes the physical mechanisms rather than the empirical structures. Thus, stars whose light curves are "shape changers" in Paper II become stars exhibiting probable spot evolution or migration here, and FGK stars whose Lomb-Scargle (LS) periodogram show two close peaks in Paper II become stars showing evidence of either latitudinal differential rotation or spot evolution here. Table 2 in Paper II provides a light-curve dictionary linking emprirical shapes to probable physical mechanisms. We will make considerable use of this light-curve taxonomy.

One of the key features of Figure 1 is the existence of a slowly rotating sequence of stars; these are the stars that roughly define the upper envelope to the period-color data for spectral types F5 to late $\mathrm{K}$. This sequence becomes more prominent in older clusters (e.g., Hyades-Douglas et al. 2016; Praesepe-Agueros et al. 2011), but has clearly begun to form at Pleiades age. Features of the slow sequence that we will discuss at length in the next section include the blue edge to the sequence at $\left(V-K_{\mathrm{s}}\right)_{0} \sim 1.1$ (Section 3.2), the red edge to the sequence at $\left(V-K_{\mathrm{s}}\right)_{0} \sim 3.7$ (Section 3.3), and an apparent kink in the sequence at $\left(V-K_{\mathrm{s}}\right)_{0} \sim 2.6$ (Section 3.4).

We initially identified stars belonging to the slow sequence by eye. To define the sequence more quantitatively, we fit a polynomial curve to those stars in the $\log P,\left(V-K_{\mathrm{s}}\right)_{0}$ plane. That first fit emphasized that the data points seemed to show a discontinuity in period at $\left(V-K_{\mathrm{s}}\right)_{0} \sim 2.6$; we therefore fit two separate curves to the data, one blueward and one redward of $\left(V-K_{\mathrm{s}}\right)_{0}=2.6$. Because nearly all of the stars we had identified by eye as belonging to the slow sequence had periods within $\pm 30 \%$ of those curves, we then used that criterion to define our final set of slow-sequence stars. The two curves and the final set of slow-sequence stars are shown in Figure 2. ${ }^{16}$ The extension of the red curve to $\left(V-K_{\mathrm{s}}\right)_{0}=5$ is obviously quite uncertain, but the exact shape is not important for the purpose for which we will use it. Finally, we have split the remaining stars with $1.1<\left(V-K_{\mathrm{s}}\right)_{0}<3.7$ into two other groups based on their periods - a set of the fastest rotators, with $P($ obs $) / P($ seq $)<0.13$, and a set of intermediate rotators, with $0.13<P(\mathrm{obs}) / P(\mathrm{seq})<0.7$, where $P(\mathrm{seq})$ is the period predicted for that $\left(V-K_{\mathrm{s}}\right)_{0}$ by the polynomial curves. The slow, intermediate, and fast rotation groups are color-coded red, green, and blue, respectively, in Figure 2. We will utilize the polynomial curves and the three rotation groups in Section 3.1.

For the $\mathrm{G}$ and $\mathrm{K}$ dwarfs in clusters older than about $600 \mathrm{Myr}$, all (or nearly all) stars are on the slow sequence (Radick et al. 1987; Stauffer et al. 1987; Agueros et al. 2011; Delorme et al. 2011). By contrast, 1-3 Myr old PMS stars of this mass show a wide range of rotational velocities (e.g., Carpenter et al. 2001; Herbst et al. 2001; Rebull et al. 2002b; RodriguezLedesma et al. 2009; Affer et al. 2013). The collapse from the wide initial range in angular momenta to a nearly unimodal distribution at a given mass by Hyades age is normally attributed to a disk-locking mechanism that temporarily inhibits spin-up for PMS stars with actively accreting disks (Ghosh \& Lamb 1979; Königl 1991, Collier Cameron et al. 1995; Bouvier et al. 2007, p. 479) and to stellar winds and an angular

\footnotetext{
16 The equation for $1.1<\left(V-K_{\mathrm{s}}\right)_{0}<2.6$ is $\log (P)=-0.8905+$ $1.3658 \times\left(V-K_{\mathrm{s}}\right)_{0}-0.2616 \times\left(V-K_{\mathrm{s}}\right)_{0}^{2}$ and for $2.6<\left(V-K_{\mathrm{s}}\right)_{0}<3.7$ is $\log (P)=-0.1604+0.4809 \times\left(V-K_{\mathrm{s}}\right)_{0}-0.0411 \times\left(V-K_{\mathrm{s}}\right)_{0}^{2}$.
} 


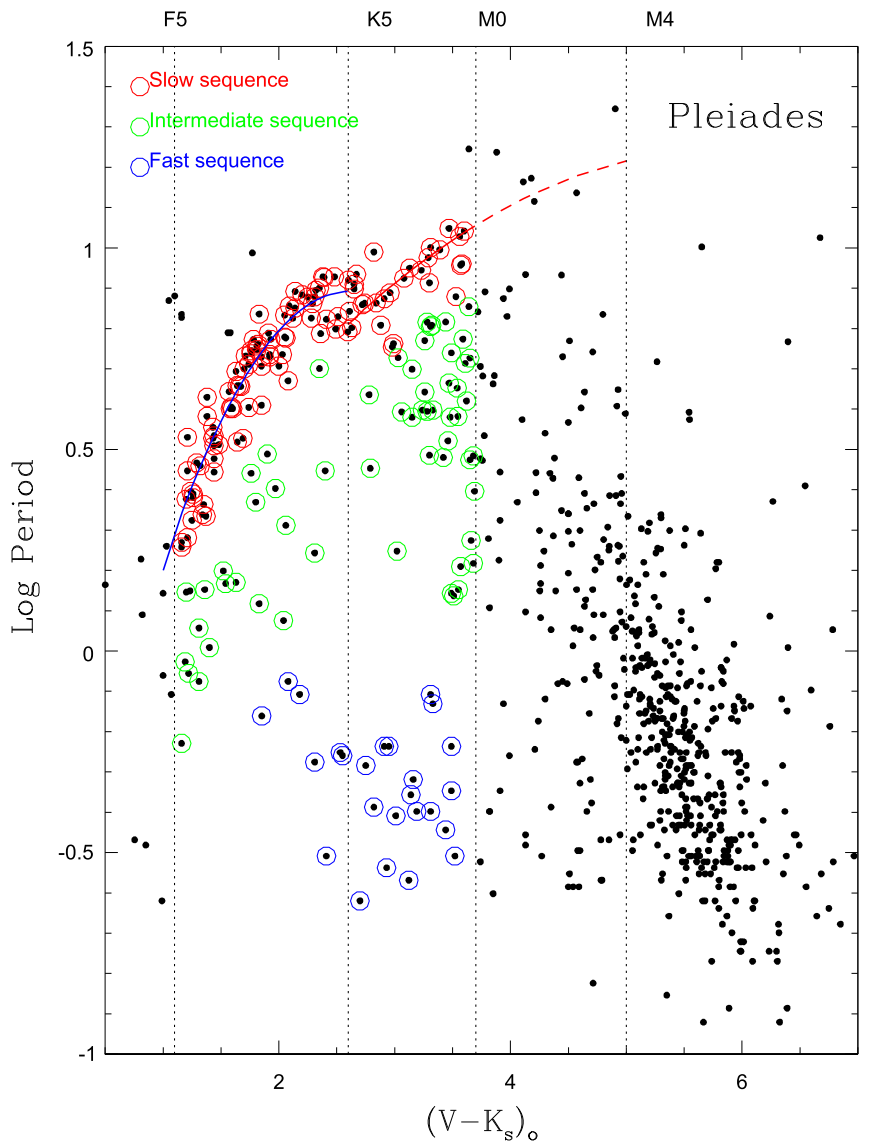

Figure 2. Kepler $K 2$ rotation periods for probable members of the Pleiades vs. their estimated $\left(V-K_{\mathrm{S}}\right)_{0}$ colors. The red, green, and blue colored circles denote, respectively, stars with $1.1<\left(V-K_{\mathrm{s}}\right)_{0}<3.7$ having slow, intermediate, and fast rotation rates (groups that we use in our analysis in later sections). The left three vertical dotted lines denote the bins in $\left(V-K_{\mathrm{s}}\right)_{0}$ color used to create the histograms in Figure 3. The fourth vertical line at $\left(V-K_{\mathrm{s}}\right)_{0}=5.0$ corresponds approximately to the point redward of which stars should be fully convective. The blue curve is a least-squares fit to the slow-sequence stars for $\left(V-K_{\mathrm{s}}\right)_{0}<2.6$; the red curve is a fit to the slowsequence stars for $2.75<\left(V-K_{\mathrm{s}}\right)_{0}<3.7$. The red curve is extrapolated to $\left(V-K_{\mathrm{s}}\right)_{0}=5.0$ in order to roughly pass through the very slowly rotating Pleiades members beyond $\left(V-K_{\mathrm{s}}\right)_{0}=3.7$ (see text and Section 5.2).

momentum loss rate that is largest for rapidly rotating stars (e.g., Skumanich 1972; Stauffer \& Hartmann 1987; Soderblom et al. 1993b; Collier Cameron \& Jianke 1994; Sills et al. 2000; Gallet \& Bouvier 2013, 2015). The distribution of rotation periods as a function of mass for a cluster where the $\mathrm{G}$ and $\mathrm{K}$ dwarfs have just arrived or are near to the ZAMS - as is the case for the Pleiades-therefore becomes an important fiducial data set with which to confront angular momentum evolutionary models. We provide one representation of this distribution here by taking the rotation periods in Figure 2, dividing those periods by the period inferred from the polynomial fits to the slow sequence, and determining the relative period distribution as a function of $\left(V-K_{\mathrm{s}}\right)_{0}$ color. These distributions are plotted as histograms in Figure 3, for the color bins indicated by the vertical dashed lines in Figure 2. The fraction of stars included in the slowly rotating sequence decreases to lower mass (redder color). Specifically, if one takes $N$ (slow) to be the number of stars within $30 \%$ of the value predicted by the polynomial curves shown in Figure 2 (corresponding to the stars circled in red in that figure) and $N$ (tot) to be the total number of stars with periods in that color
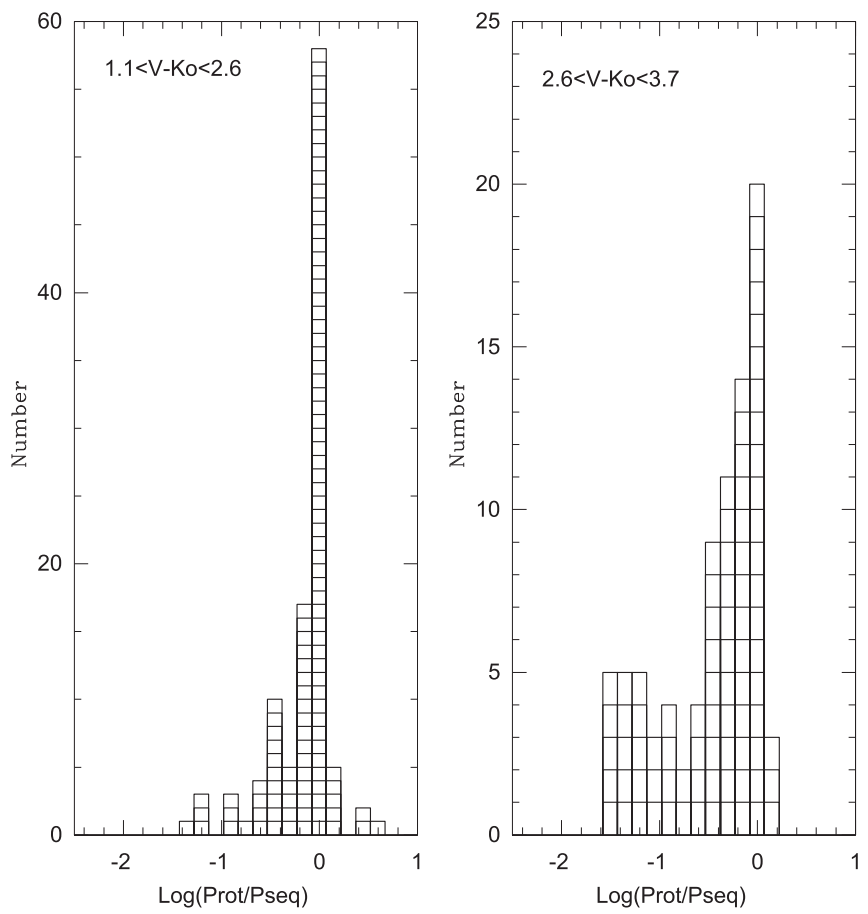

Figure 3. Histograms of the Pleiades rotation period distribution for stars with $1.1<\left(V-K_{\mathrm{s}}\right)_{0}<2.6$ and for $2.6<\left(V-K_{\mathrm{s}}\right)_{0}<3.7$. The histograms were created by taking the ratio of the observed period to the period at that color for the polynomial fits to the slow-sequence stars. Two-thirds of the stars in the bluer interval (left panel) have periods within $30 \%$ of the polynomial fit vs. only one-third for the stars in the redder color interval (right panel).

bin, the ratio of $N$ (slow) to $N$ (tot) is $0.69,0.32$, and 0.02 for the color intervals1.1 $<\left(V-K_{\mathrm{s}}\right)_{0}<2.6,2.6<\left(V-K_{\mathrm{s}}\right)_{0}<3.7$, and $3.7<\left(V-K_{\mathrm{s}}\right)_{0} 5.0$, respectively.

\section{THE ROTATION PATTERNS OF 0.6-1.1 $M_{\odot}$ STARS AT PLEIADES AGE}

We split our discussion of the Pleiades $K 2$ data into two main color (or mass) groupings. Here we discuss stars with $1.1<\left(V-K_{\mathrm{s}}\right)_{0}<3.6$, roughly corresponding to a mass range of 0.6-1.1 $M_{\odot}$. In Section 4, we discuss M dwarfs, corresponding to masses $<0.6 M_{\odot}$.

\subsection{Why Are Some Pleiades FGK Stars So Slow?}

At constant mass, the $\mathrm{K}$ dwarfs in the Pleiades show a range of $\sim 40$ in their observed rotation periods (i.e., from $\sim 0.25$ days to $\sim 10$ days), and hence a similar range in their inferred total angular momentum assuming solid-body rotation. Understanding why this very large dispersion in rotational histories exists has obvious importance for understanding both star and planet formation. Theoretical models for angular momentum evolution (e.g., Gallet \& Bouvier 2013, 2015) can account for this large range in rotation at a given mass by adjusting parameters such as the disk-locking timescale, core-envelope coupling timescale, and wind saturation velocities. However, it is possible that other mechanisms may also contribute. Binarity is one obvious potential mechanism for affecting the angular momenta of ZAMS-age stars because binary companions have at least the potential to truncate circumstellar disks and reduce the efficacy of PMS disk locking (Patience et al. 2002; Meibom et al. 2007). In order to test this idea, we have identified a sample of photometric binaries among the $K 2$ Pleiades sample 


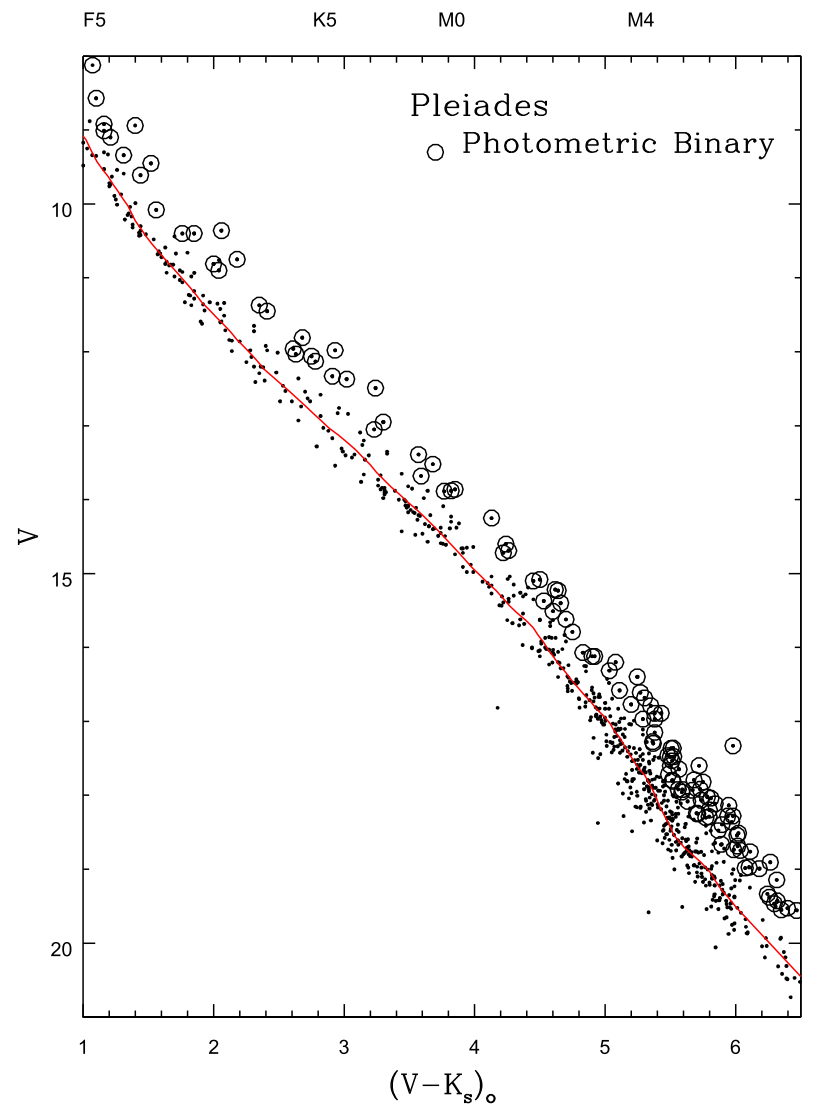

Figure 4. CMD showing the Pleiades members for which we have derived rotation periods, marking the stars identified by us as photometric binaries with circles.

using the $V$ versus $V-I_{\mathrm{C}}$ and $V$ versus $\left(V-K_{\mathrm{s}}\right)_{0}$ colormagnitude diagrams (CMDs). In the former (which uses photometry from Stauffer et al. 1987 and references therein, and from Kamai et al. 2014), we identify stars as photometric binaries if they lie more than 0.3 mag above a single-star mainsequence locus. For the latter, using the estimates of $\left(V-K_{\mathrm{s}}\right)_{0}$ as described in Paper I, we adopt $\Delta V=0.45 \mathrm{mag}$ for $\left(V-K_{\mathrm{s}}\right)_{0}<5$ and $\Delta V=0.55 \mathrm{mag}$ for $\left(V-K_{\mathrm{s}}\right)_{0}>5$ to identify probable photometric binaries. The required $\Delta V$ is larger for the $\left(V-K_{\mathrm{s}}\right)_{0} \mathrm{CMD}$ because of the lower accuracy of the (less homogeneous) $\left(V-K_{\mathrm{s}}\right)_{0}$ data; the limit changes at $\left(V-K_{\mathrm{s}}\right)_{0}=5$ because the slope of the single-star locus increases at about that color. Stars selected as binaries from the $\left(V-K_{\mathrm{s}}\right)_{0} \mathrm{CMD}$ are illustrated in Figure 4 . The advantage of this purely photometric selection technique is that we can apply it for every star for which we have $K 2$ rotation period data.

We replot the period-color data for the Pleiades FGK stars (roughly stars with $\left.1.1<\left(V-K_{\mathrm{s}}\right)_{0}<3.7\right)$ in Figure 5, where the photometric binaries we have identified are now shown as diamonds. This diagram shows that members of the slow sequence are infrequently identified as photometric binaries ( 9 photometric binaries out of 105 total), whereas the stars with intermediate rotation periods are photometric binaries with significantly higher frequency. In Paper I (Section 3.2, Figure 11), we show that more than a dozen stars in the slowly rotating sequence have been identified as spectroscopic binaries. Nearly all are single-line spectroscopic binaries, consistent with many of them not having been identified by us as photometric binaries. Our association of being a

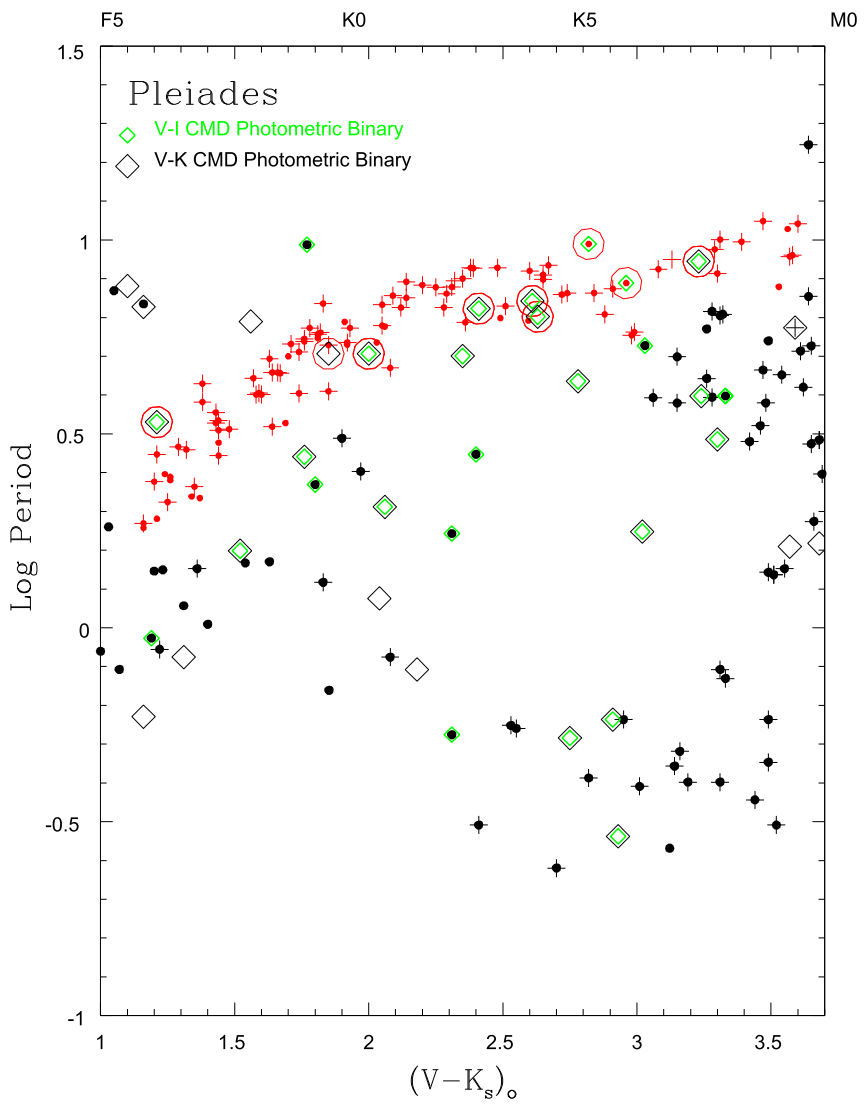

Figure 5. Period-color plot, marking photometric binaries. Dots and plus signs are photometric singles, based on the $\left(V-K_{\mathrm{s}}\right)_{0}$ and $\left(V-I_{\mathrm{c}}\right)_{0}$ CMDs, respectively. They are colored red if on the slow sequence and black otherwise. Photometric binaries are shown as diamonds, in green if based on the $\left(V-I_{\mathrm{c}}\right)_{0}$ CMD and in black if based on the $\left(V-K_{\mathrm{s}}\right)_{0}$ CMD. Photometric binaries that are on the slow sequence are additionally marked by large red circles. Given the differing sensitivities of the two CMDs to binaries, the sorting into single and binary classes is typically quite consistent. Members of the slowly rotating sequence are seldomly photometric binaries.

photometric binary and having somewhat more rapid rotation could be reconciled with these additional spectroscopic binaries in the slow sequence if the physical mechanism leading to relatively rapid rotation operates most efficiently for nearly equal mass systems having relatively large semimajor axes.

In order to illustrate this same point in a more quantitative way, we have calculated "vertical" displacements of each star from the single-star $V$ versus $\left(V-I_{\mathrm{c}}\right)_{0}$ locus $(d V)$ and normalized period displacements from the slow-sequence locus curves $\left(d P / P_{\mathrm{seq}}\right)$-where $P_{\mathrm{seq}}$ is the period predicted by the polynomial fits - and plot those two indices in Figure 6. We use $\left(V-I_{\mathrm{c}}\right)_{0}$ rather than $\left(V-K_{\mathrm{s}}\right)_{0}$ for this purpose because the $\left(V-I_{\mathrm{c}}\right)_{0}$ photometry is more accurate, it is less affected by spottedness (Stauffer et al. 2003), and in this magnitude range we have published $\left(V-I_{\mathrm{c}}\right)_{0}$ photometry for more than threequarters of the Pleiades members (Stauffer et al. 1987 and references therein; Kamai et al. 2014). Figure 6 shows that the stars with intermediate rotation rates are much more frequently displaced significantly above the single-star CMD locus than either of the other two groups. The fraction of stars more than 0.3 mag above the single-star locus is $10 \%$ for the slow sequence, $41 \%$ for the intermediate sequence, and $14 \%$ for the fast sequence. Collapsing the distribution in the other axis, $62 \%$ (18 of 29) of the stars identified as photometric binaries versus 


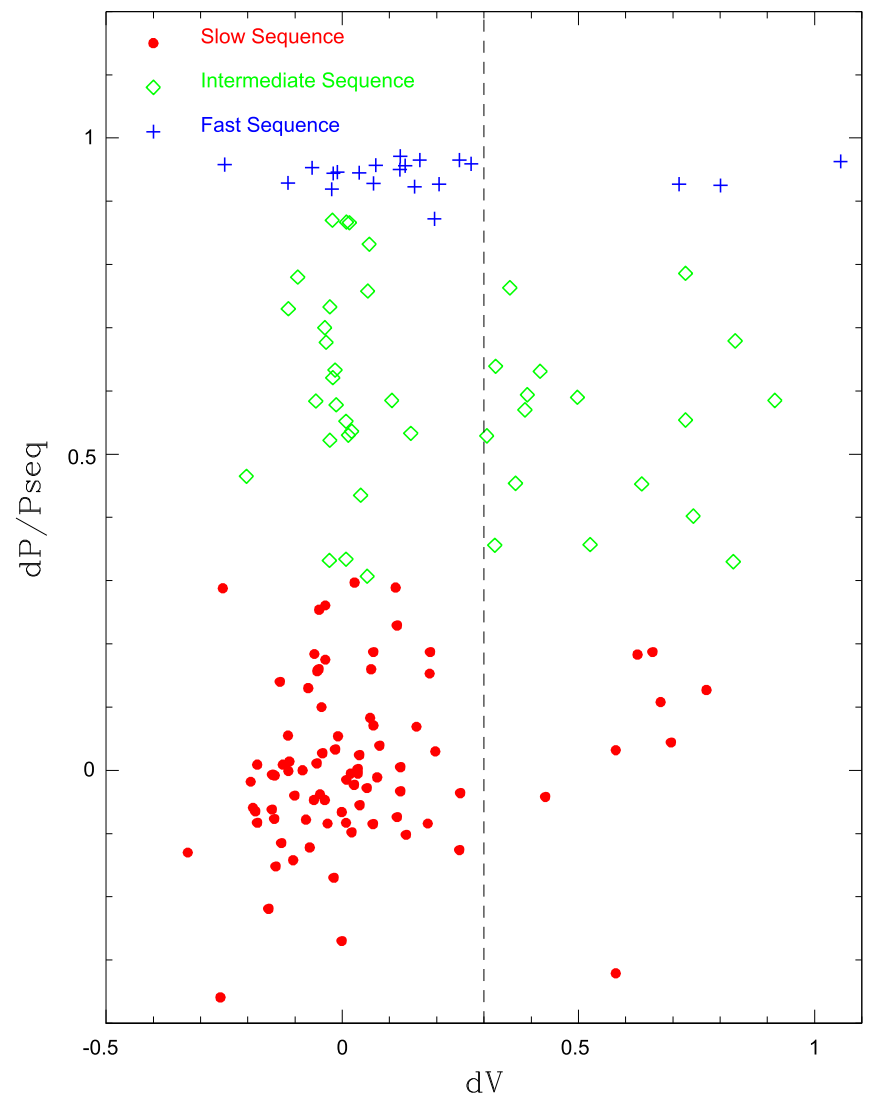

Figure 6. Correlation between the displacement of stars above the mainsequence locus curve in the $V$ vs. $V-I_{\mathrm{C}} \mathrm{CMD}(d V)$ and a measure of how much the star's rotation period differs from the slow-sequence locus curves. The $y$-axis is $d P / P_{\text {seq }}=\left(P_{\text {seq }}-P_{\text {rot }}\right) / P_{\text {seq }}$, where $P_{\text {seq }}$ is the period at that color for the curves shown in Figure 2. Stars more rapidly rotating than the slowsequence locus have positive values of $d P / P_{\text {seq }}$; stars displaced above the single-star locus have positive values of $d V$. The plot demonstrates that a large fraction of the stars with intermediate rotational velocities are likely photometric binaries. One star, HII 303, has $\Delta V>1.1$ and does not appear; it is a member of the intermediate rotation group and has been identified as at least a triple system (Bouvier et al. 1997). HII 3197, the fast rotator at $\Delta V=1.05$, was also identified as a triple by Bouvier et al. (1997).

$22 \%(26 / 120)$ of the single stars are members of the intermediate rotation group. Just from binomial statistics, the probability that so many of the objects in the intermediate rotation group are photometric binaries is less than 1 part in 1000 , while the probability that so few of the objects in the slowly rotating group are photometric binaries is of order 1 in 100. We therefore conclude that there is very likely a real link between rotation rate at Pleiades age in this mass range and having a comparable mass companion. A plausible but not unique physical explanation for this correlation is that binary stars with some range of parameters (mass ratio and separation presumably being most important) inhibit disk-dependent PMS angular momentum regulation mechanisms. ${ }^{17}$

If the correlation between binarity and rotation rate arises due to the effect of binarity on the lifetime or amount of dust in

\footnotetext{
17 This tendency for young GK binaries to be more rapidly rotating than their isolated counterparts could possibly explain a long-standing mystery in the Hyades. Pye et al. (1994) showed that the K dwarf binaries in the Hyades have, on average, much larger $\mathrm{X}$-ray luminosities than the single $\mathrm{K}$ dwarfs in the Hyades. Those binaries have long orbital periods, so tidal spin-up is not an answer. If our Pleiades conjecture is correct, the Hyades $\mathrm{K}$ dwarf binaries could be more rapidly rotating than their single counterparts, thereby leading to their excess X-ray luminosities.
}

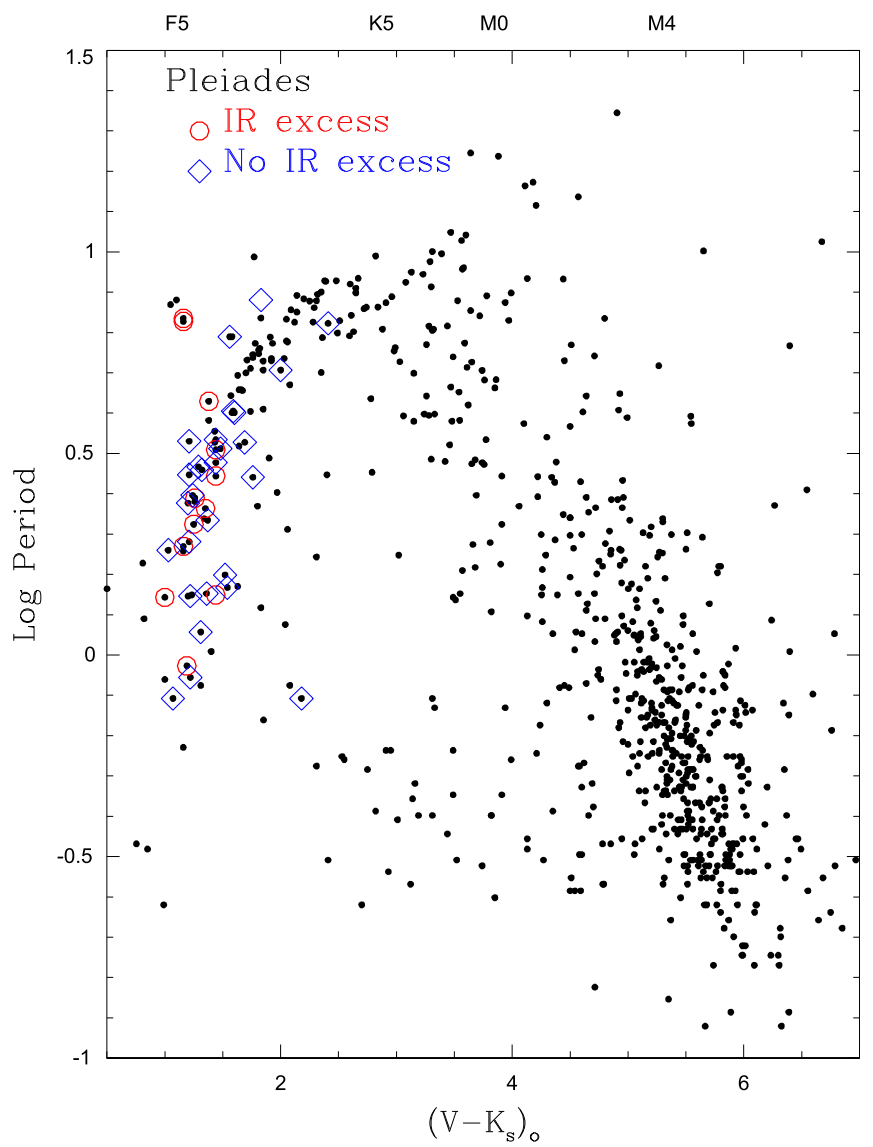

Figure 7. Period-color plot, marking stars with debris disks from Sierchio et al. (2010). In the color range where there are good Spitzer $24 \mu \mathrm{m}$ data, essentially all the stars are on or near the slowly rotating sequence. Determining whether debris disks and stellar rotation correlate at Pleiades age requires new IR data for lower-mass stars.

the inner disk during PMS evolution, it is possible that this effect could be reflected in the presence or strength of debris disk signatures at later epochs. A number of models predict that the rotation period of low-mass PMS stars will be locked to the Keplerian rotation period of their inner circumstellar disk (Königl 1991; Bouvier et al. 2007, p. 479). This suggests the possibility that the stars with the longest-lived PMS circumstellar disks might arrive on the main sequence as slow rotators (e.g., Bouvier et al. 1997). If those long-lived disks generate more planets and planetessimals, then it is possible that their signature at later epochs could be more dust from planetessimal collisions, which would be detected in the mid- to far-infrared as debris disks. We might then expect a correlation where the stars in the slowly rotating sequence are also frequently identified as having detectable debris disks. Such a correlation was noted by Sierchio et al. (2010), but the statistical significance was meager, in part because only $v \sin i$ data were available for many of the stars at that time. In Figure 7, we again replot the $K 2$ period-color data for the Pleiades, this time highlighting stars with detected debris disks as red circles. Unfortunately, in the color range where there are detections (basically spectral type $F$ and early $G$ ), there is no clear separation between a slowly and rapidly rotating sequence, and therefore (in agreement with Sierchio et al.) we cannot draw any conclusions concerning a possible correlation of debris disks and rotation. The stars of interest are too faint for WISE; a test of the hypothesized correlation between debris disk IR 
excess and rotation must await a more sensitive set of far-IR data, extending into the late $G$ and $K$ spectral types in the Pleiades.

\subsection{The Blue Edge-At What Color Do Nonaxisymmetrically Distributed Spots First Appear?}

High-resolution spectra obtained more than 50 yr ago (Anderson et al. 1966) showed that, on average, rotational velocities for Pleiades members more massive than the Sun decrease rapidly going from early to late $\mathrm{F}$. Improved $v \sin i$ for Pleiades F stars were later obtained by Soderblom et al. (1993a) and Queloz et al. (1998). Those data showed mean rotation rates decreasing from about $50 \mathrm{~km} \mathrm{~s}^{-1}$ at F2 to about $15 \mathrm{~km} \mathrm{~s}^{-1}$ at G0. Therefore, the most rapidly rotating Pleiades $\mathrm{F}$ dwarfs are at the earliest subtypes. However, because early F dwarfs are not expected to have outer convective envelopes, they should not have magnetic dynamos or solar-like starspots. Without spots, these stars should not have rotationally driven photometric variability, and hence they should not appear in our $K 2$ period catalog. Outer convective envelopes of sufficient depth to generate significant energy from a magnetic dynamo are believed to first appear around spectral type F5 (Wilson 1966), so it is near that spectral type where one might expect to identify spot-induced rotational variability. Our $K 2$ data should allow us to accurately determine that transition point. Unfortunately, ZAMS F stars also occupy a $T_{\text {eff }}$ range where pulsation is expected to generate periodic photometric variability (Dziembowski 1977; Dupret et al. 2004). In the late $\mathrm{A}$ to early $\mathrm{F}$ spectral range, one expects to find $\delta$ Sct pulsators; for somewhat cooler stars-spectral types generally about F0-F3 - one expects $\gamma$ Dor type pulsation (Balona et al. 2011). From the perspective of this paper, it is fortunate that the $\delta$ Sct pulsators have typical periods of $0.03-0.3$ days (Balona et al. 2015), allowing their pulsational variability to be easily distinguished from the rotational modulation of $\mathrm{F}$ stars, where one expects periods greater than 0.5 days (also see Paper II).

$\gamma$ Dor variables, on the other hand, are expected to have dominant periods of order 0.4-3.0 days (Kaye et al. 1999), placing their pulsation range directly in the range of rotation periods expected for ZAMS-age F dwarfs. Indeed, while Balona et al. (2011) identify several hundred stars with light curves in the main Kepler field as probable $\gamma$ Dor variables, they also acknowledge that the variability of many of those stars may in fact be due to rotational modulation and starspots. For our purposes, we choose to avoid this issue by relying on the amplitude of variability (as determined in Paper I) to define the boundary redward of which we are reasonably certain that spots dominate the variability (and the periods we find are the star's rotation period). Figure 8 shows the variation of the amplitude of photometric variability for stars where we determine periods versus $\left(V-K_{\mathrm{s}}\right)_{0}$ color. There is a fairly sharp boundary at $\left(V-K_{\mathrm{s}}\right)_{0} \sim 1.1$, redward of which the amplitudes become much larger; the spectral type corresponding to that color (from Pecaut \& Mamajek 2013, Table 5) is $\mathrm{F} 5$, the same boundary where one begins to see significant chromospheric emission. Immediately blueward of that boundary, we believe it is entirely possible that much of the variability seen in our $K 2$ light curves is rotational modulation due to small (possibly transient) magnetic spots, but separating that variability from pulsational signatures is not simple and should be the subject of a dedicated paper. From analysis of Q3 Kepler data for active stars, Reinhold et al. (2013) also

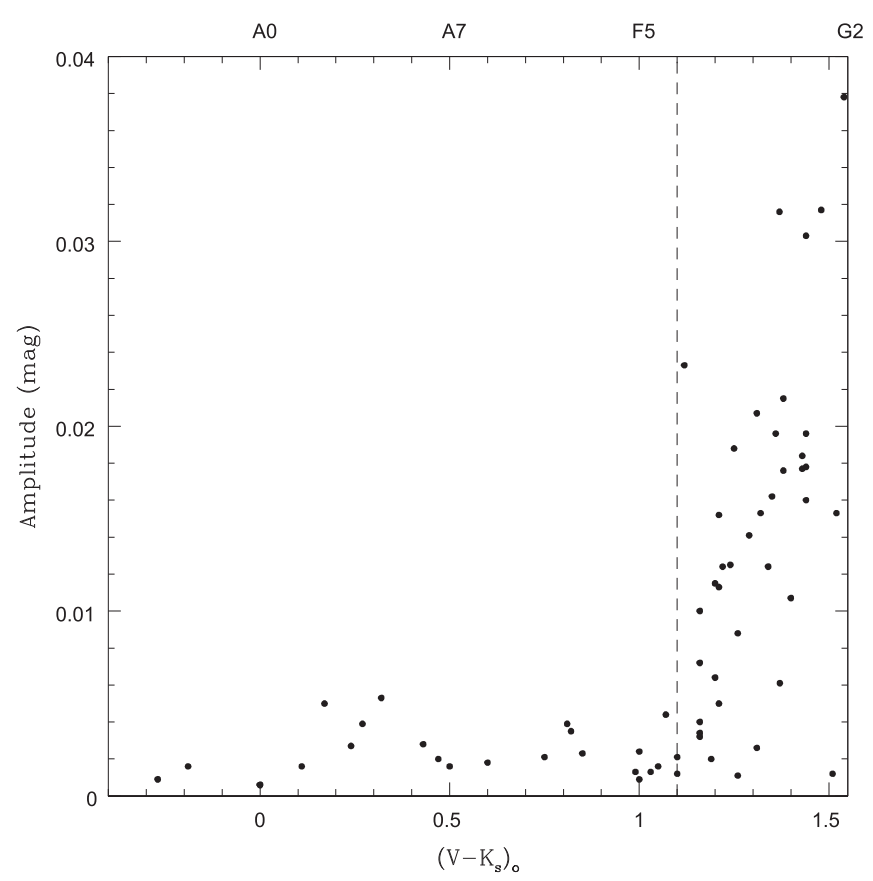

Figure 8. Light-curve amplitude, as defined in Paper I, vs. $\left(V-K_{\mathrm{s}}\right)_{0}$ color for the $\mathrm{A}$ and $\mathrm{F}$ stars in the Pleiades where we have identified at least one significant period in the LS periodogram. The vertical dashed line marks the boundary we have adopted as the blue edge of the region, where rotational modulation by magnetic spots is assumed to drive the observed photometric variability in our $K 2$ Pleiades sample. See the text for a discussion.

concluded that spectral type F5 marked a dividing line, blueward of which it was much more difficult to separate rotational variability from pulsational variability. ${ }^{18}$

\subsection{The Red Edge of the Slowly Rotating Sequence}

There is a clearly defined slow-rotation sequence in our $K 2$ Pleiades data, running from $\left(V-K_{\mathrm{s}}\right)_{0} \sim 1.1$, or spectral type F5 (see discussion in the preceding section), all the way to $\left(V-K_{\mathrm{s}}\right)_{0} \sim 3.7$, or spectral type $\mathrm{K} 8$. The mean rotation period increases from $P \sim 2$ days at the blue edge to $P \sim 11$ days at the red edge. Is the relatively sharp cutoff to the slow sequence at $\left(V-K_{\mathrm{s}}\right)_{0} \sim 3.7$ simply a direct and readily measureable indicator of the cluster's age, or is there some other physical mechanism that contributes to this sharp end to the slowly rotating sequence at this particular age? We suspect that the former answer is mostly correct, and we provide one figure that strongly supports this view; however, we also offer one (possibly coincidental) piece of evidence in favor of the latter idea.

Direct evidence that the location of the red edge for the slowly rotating sequence is a signpost of the age for a coeval population of stars comes from comparison of the Pleiades $K 2$ rotation period data to similarly processed and analyzed $K 2$ Field 5 data for the much older ( 600-800 Myr) Praesepe cluster. We have conducted a quick-look analysis of the publicly available $K 2$ Field 5 light curves for Praesepe for the sole purpose of making the comparison to the Pleiades data.

\footnotetext{
18 As shown later in Figure $9,\left(V-K_{\mathrm{s}}\right)_{0}=1.1$ is also approximately the color where the slow-sequence locus curve for Praesepe (age 600-800 Myr; Brandt \& Huang 2015) overlaps with the Pleiades slow locus curve. That intersection presumably marks the point where main-sequence angular momentum loss rates become negligible, further reinforcing the conclusion that this is where convection-driven magnetic dynamos become important.
} 


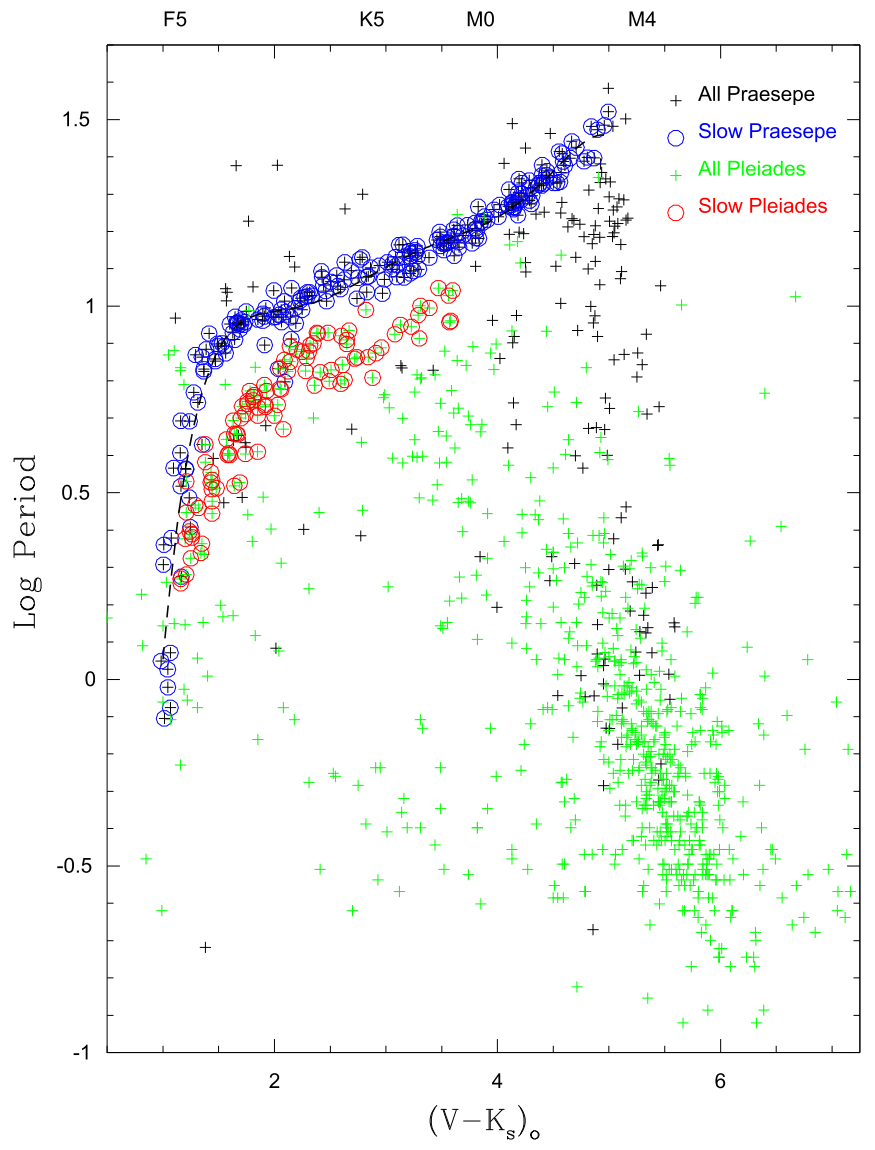

Figure 9. Kepler $K 2$ rotation periods for probable members of the Pleiades compared to $K 2$ rotation periods for Praesepe. The slow sequence evolves to longer period —and extends much redder — by Praesepe age ( $600-800 \mathrm{Myr})$, compared to the Pleiades sequence.

Figure 9 overplots the period data for Praesepe versus that for the Pleiades; the solid curve is a polynomial fit to the welldefined Praesepe slow sequence. ${ }^{19}$ Looking at the slow sequences for the two clusters near $\left(V-K_{\mathrm{s}}\right)_{0} \sim 3.5$, it is obvious that the slow sequence extends much further to the red at $700 \mathrm{Myr}$ than at $125 \mathrm{Myr}$, and therefore the location of this edge is at least partially a measure of the age of the cluster. A well-defined slow sequence at 500-800 Myr extending to early $\mathrm{M}$ is also quite evident in the ground-based rotation studies of Praesepe (Agueros et al. 2011), as well as M37 (Hartman et al. 2009) and the Hyades (Delorme et al. 2011).

While Figure 9 is quite persuasive, it is nevertheless true that the red edge in the Pleiades occurs almost precisely at the boundary between spectral types $\mathrm{K}$ and $\mathrm{M}$. That boundary corresponds approximately to the point where molecules become prevalent in the photospheres of low-mass mainsequence stars, and hence at the point where free electrons become less prevalent (Mould 1976). This point is illustrated in a $J-H$ versus $H-K_{s}$ color-color diagram on the $K 2$ Pleiades stars (Figure 10), where we have highlighted the stars in the slow sequence. It is apparent that the slow sequence in the Pleiades ends almost exactly where the dwarf sequence

\footnotetext{
19 The equation for the polynomial fit to the Praesepe slow sequence is $\log P=-13.190+30.079 \times\left(V-K_{\mathrm{s}}\right)_{0}-25.9416 \times\left(V-K_{\mathrm{s}}\right)_{0}^{2}+$ $11.59469 \times\left(V-K_{\mathrm{s}}\right)_{0}^{3}-2.82725 \times\left(V-K_{\mathrm{s}}\right)_{0}^{4}+0.35736 \times\left(V-K_{\mathrm{s}}\right)_{0}^{5}-$ $0.018320 \times\left(V-K_{\mathrm{s}}\right)_{0}^{6}$.
}

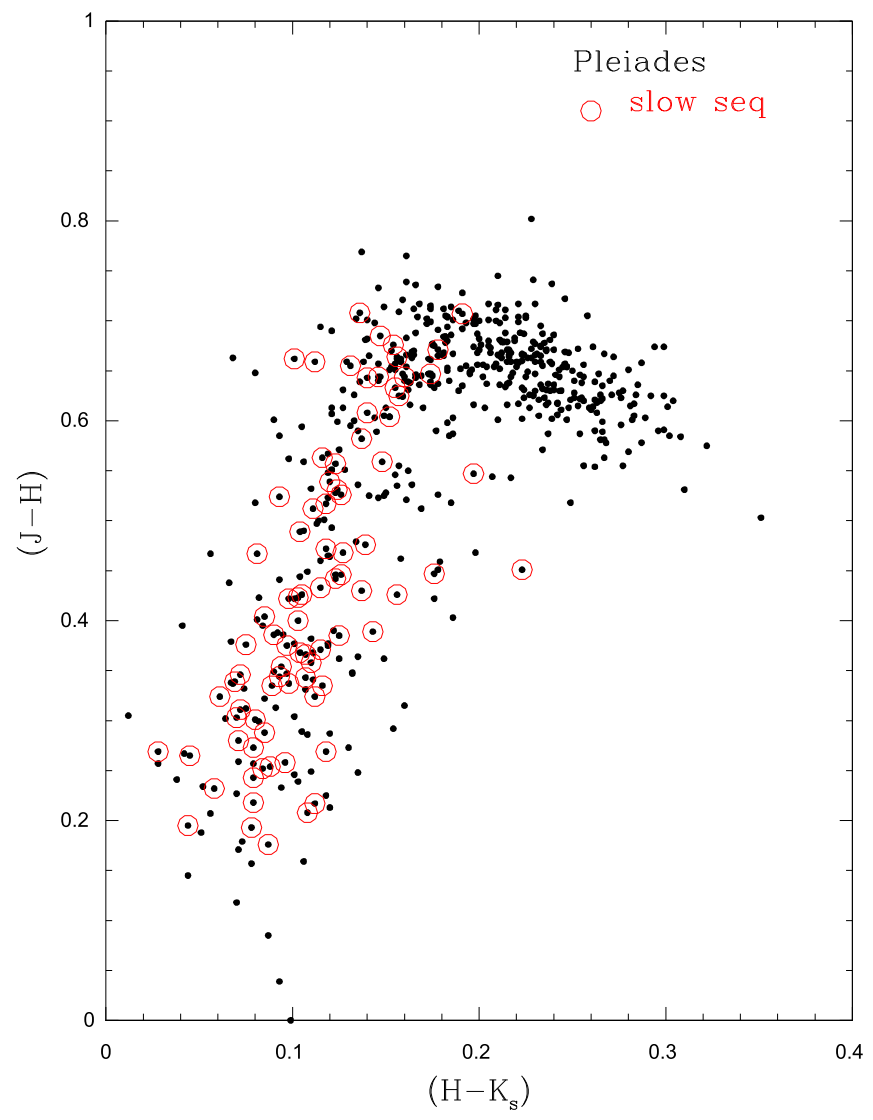

Figure 10. $J-H$ vs. $H-K_{s}$ color-color diagram for Pleiades stars with $K 2$ rotation periods, highlighting members of the slowly rotating sequence. The coolest slow-sequence stars fall just at the mass where $J-H$ stops getting redder to lower mass, signaling the reduction in $\mathrm{H}^{-}$opacity and the ramp-up of molecule opacities for lower masses.

colors turn over in the $J-H$ versus $H-K_{s}$ diagram (i.e., where $J-H$ stops getting redder as $T_{\text {eff }}$ decreases and instead slowly becomes bluer with decreasing $\left.T_{\text {eff }}\right)$. It could be that it is simply coincidental that the red limit to the slow sequence happens to occur at Pleiades age at exactly this color. Or, it may be that there is a (possibly small) decrease in the average angular momentum loss rate at this color as a result of the reduced ionization fraction in the photosphere, such that the red limit to the slow sequence stalls at this color for a time rather than increasing more or less linearly with age. We will return to this topic at the end of the next section.

\subsection{Is the Kink in the Slow Sequence at $V-K \sim 2.6$ Real? Is It Present in Other Young Clusters?}

We noted a kink at $\left(V-K_{\mathrm{s}}\right)_{0} \sim 2.6$ in the slowly rotating Pleiades sequence in Section 2. We highlight that kink here in Figure 11 with an expanded plot of just the relevant portion of the $\log P$ versus $\left(V-K_{\mathrm{s}}\right)_{0}$ diagram, which again compares stars in the Pleiades and Praesepe. The curves are the secondorder polynomial fits to the Pleiades slow-sequence stars described in Section 2. There is obviously no kink in the Praesepe slow sequence near $\left(V-K_{\mathrm{S}}\right)_{0}=2.6$, but the kink seems fairly evident in the Pleiades. ${ }^{20}$ This kink in the period-

\footnotetext{
${ }^{20}$ The kink is also visible in Figure 12 of Hartman et al. (2010) at $M_{K} \sim 4.7$, using ground-based periods for the Pleiades. Our figure and theirs share many of the same stars, so this does not provide significant confirmation of the existence of the kink.
} 


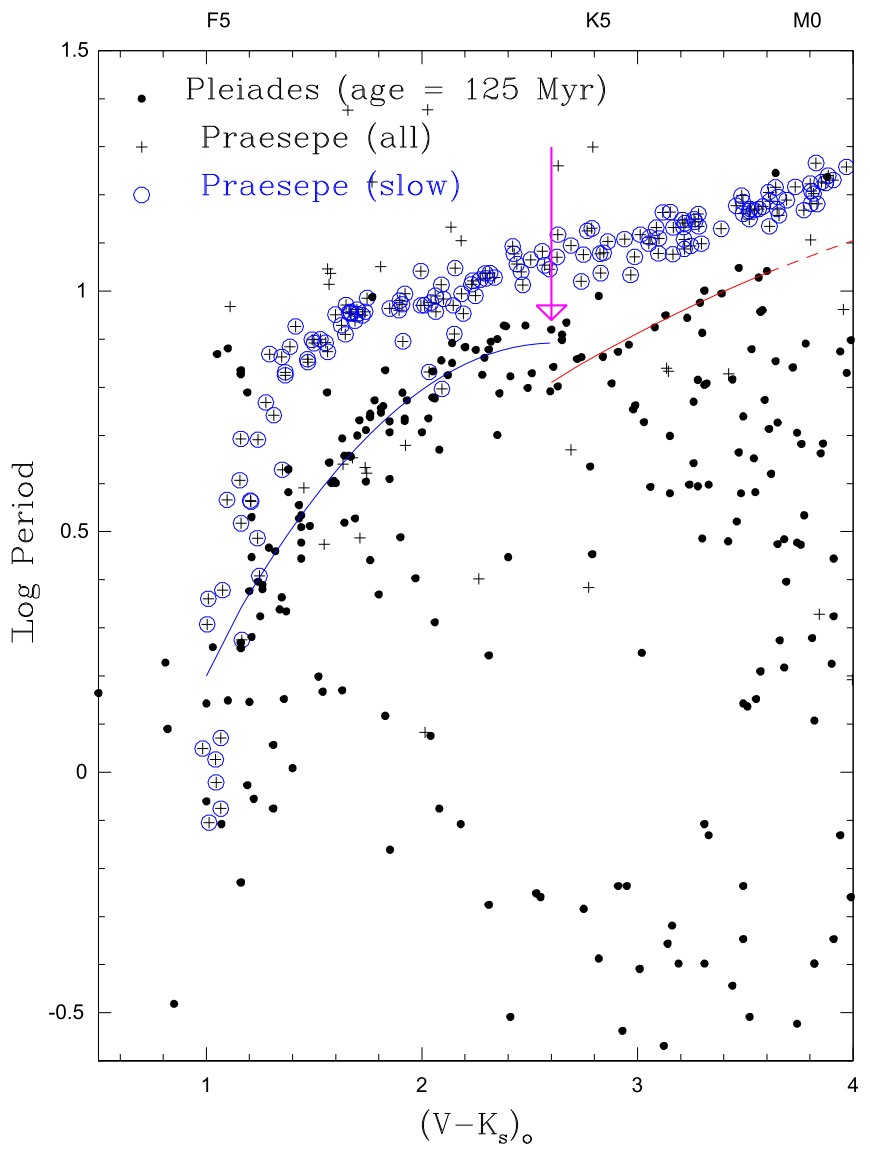

Figure 11. Blow-up of the $\log P$ vs. $\left(V-K_{\mathrm{s}}\right)_{0}$ diagram for the Pleiades and Praesepe, highlighting the region of color-period space inhabited by the slowly rotating sequence of stars in the Pleiades. The plot illustrates that (a) the Pleiades slow sequence overlaps with the Praesepe slow sequence at about $\left(V-K_{\mathrm{s}}\right)_{0}=1.1$, and hence that color marks where significant main-sequence angular momentum loss from winds begins; (b) the two sequences have maximum separation among the G dwarfs; (c) the separation between the two sequences reaches a minimum at about $\left(V-K_{\mathrm{s}}\right)_{0}=2.3$; (d) there is a discontinuity (a kink) in the Pleiades slow sequence at $\left(V-K_{\mathrm{s}}\right)_{0} \sim 2.6$; and (e) the Pleiades slow sequence ends at $\left(V-K_{\mathrm{s}}\right)_{0} \sim 3.7$ and $P=11.0$ days (vs. $P=15.0$ days in Praesepe at that color). The magenta arrow marks the location of the kink in the Pleiades slow sequence.

color distribution could simply be illusory and attributable to small number statistics. However, data exist both in other clusters and within our $K 2$ Pleiades observations that suggest that the kink may be real; we describe these data now.

The presence of a kink in the slow sequence at Pleiades age ( $125 \mathrm{Myr})$ but not at Praesepe age ( $\sim 600-800 \mathrm{Myr})$ suggests that this could be an age-related phenomenon. If so, other clusters of similar age to the Pleiades should also show the kink. Large samples of rotation periods have now been published for many open clusters (e.g., Irwin et al. 2007, 2008, 2009; Messina et al. 2010; Barnes et al. 2015). However, in many cases the exposure times and areal coverage for those surveys are such that $M$ dwarfs are well sampled in the data but relatively few $\mathrm{K}$ dwarfs are included, making those data unsuitable for our purposes. Two relatively young clusters for which rotation periods have been published with good coverage of the $\mathrm{K}$ dwarf regime do exist, though; these two clusters are M35 (age 160 Myr) and M34 (age 220 Myr). Figure 12 compares the rotation period distribution for those
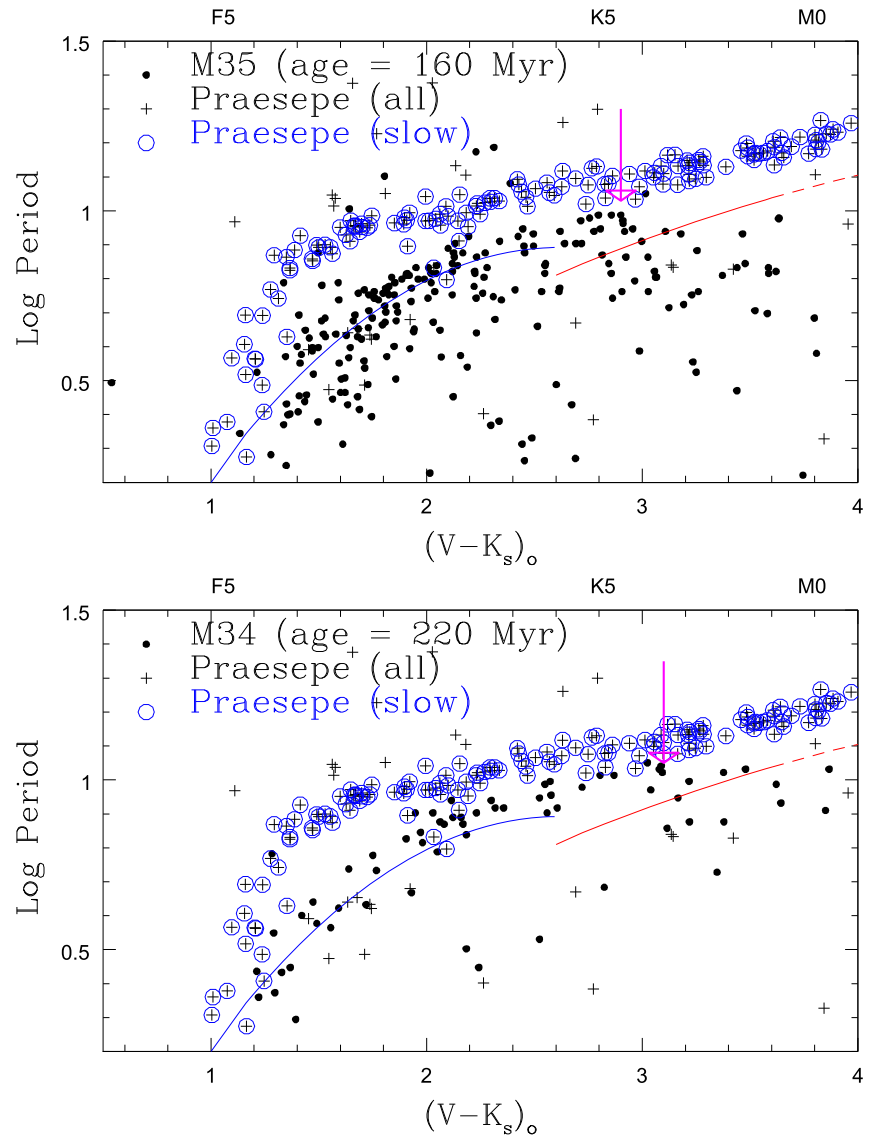

Figure 12. Same as for Figure 11, except this time comparing ground-based period data for M35 (age = $160 \mathrm{Myr}$; Meibom et al. 2009) and M34 (age $=220 \mathrm{Myr}$; Meibom et al. 2011) to Praesepe. The two curves are the fits to the Pleiades slow-sequence stars. The M35 periods are not definitely displaced relative to the Pleiades except near spectral type K5, where the separation between the M35 and Praesepe sequences reaches a minimum. The M34 figure demonstrates well that M34 has an age intermediate between that for the Pleiades and Praesepe because the M34 slowly rotating locus falls above the Pleiades curves and below the Praesepe stars-though there is some overlap between stars in Praesepe and stars in M34 in the K dwarf mass range (illustrating the stastical nature of ages inferred from rotation). There are possible kinks (marked by magenta arrows) in the M34 and M35 slow sequences at $\left(V-K_{\mathrm{s}}\right)_{0}=3.1$ and 2.9 , respectively, similar to the kink in the Pleiades distribution at $\left(V-K_{\mathrm{s}}\right)_{0}=2.6$. See text for a discussion.

two clusters to Praesepe's rotation data, where the rotation data for these clusters come from ground-based campaigns reported in Meibom et al. $(2009,2011)$. Close examination of those plots shows the following:

1. The M35 slow sequence is very similar to the Pleiades slow sequence (as previously noted by Hartman et al. 2010). The main difference relative to the Pleiades is that the feature that could be interpreted as a kink occurs at a color significantly redder than for the Pleiades, at $\left(V-K_{\mathrm{s}}\right)_{0} \sim 2.9$.

2. The M34 slow sequence is exactly as expected, with a locus that is between that of the younger Pleiades and older Praesepe. Its slow sequence has what may be a similar kink to the Pleiades, but at a redder color than either the Pleiades or M34 $\left(\left(V-K_{\mathrm{s}}\right)_{0} \sim 3.1\right)$, with a few stars redder than those which loosely follow the lower branch of the Pleiades slow locus curve.

A logarithmic scale can sometimes be misleading and can underemphasize real but small effects. In Figure 13, we replot a 

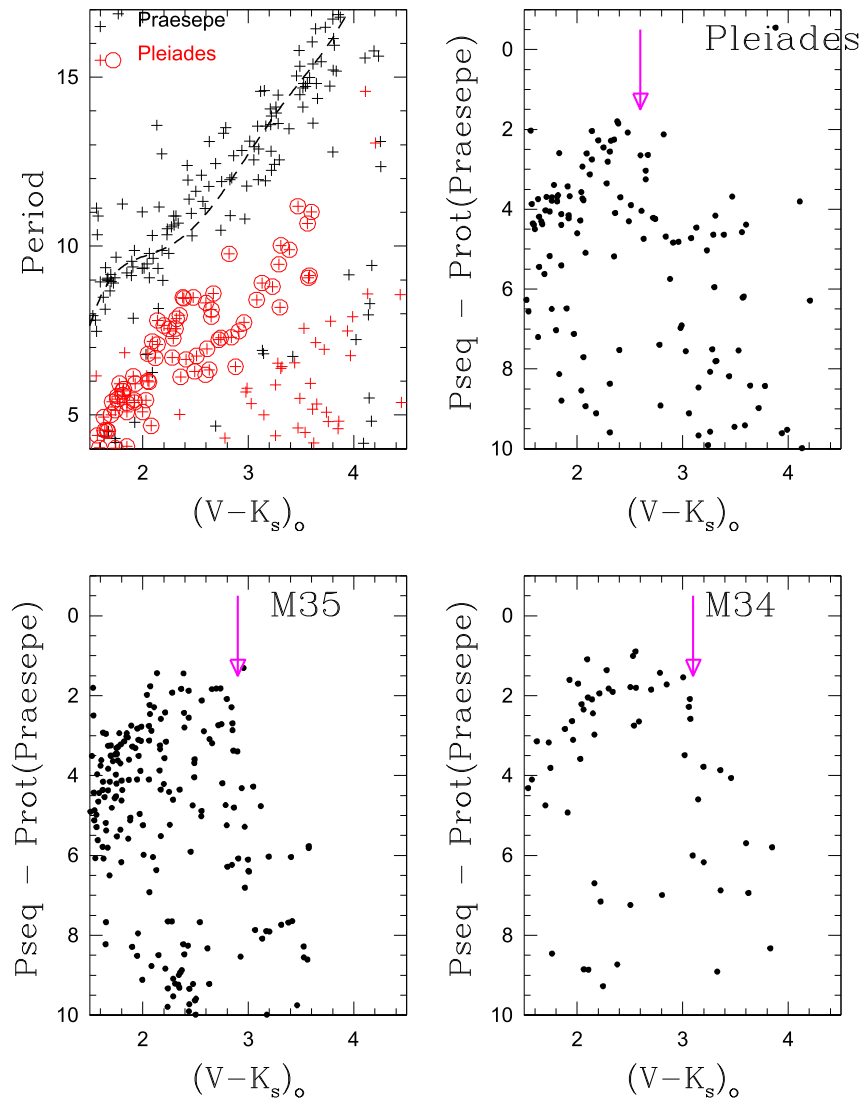

Figure 13. Top left: comparison of rotation periods in the Pleiades and Praesepe, showing just the more slowly rotation FGK stars, with the $y$-axis now being linear, with period in days. The stars in the slow rotator sequence of the Pleiades are circled in red. Top right: the same Pleiades data, also with a linear $y$-axis, but this time plotting the difference between the observed period and the period for a star of that color having the nominal slow-sequence period in Praesepe. Bottom left: same as for the previous plot, except for M35. Bottom right: same as for the previous plot, except for M34. The magenta arrows mark the $\left(V-K_{\mathrm{s}}\right)_{0}$ color of the "kink" discussed in the text; the color of the kink gets redder in step with the age of the cluster.

portion of the period data for the young clusters with a linear $y$ axis. The top left panel of Figure 13 shows the Pleiades and Praesepe stars, zooming in on the region around $\left(V-K_{\mathrm{s}}\right)_{0}=2.6$. For the other three panels of Figure 13, for each cluster we plot the difference in period between a star's observed period and the period appropriate to that color for a star in Praesepe (using the polynomial fit to the Praesepe period data shown in the first panel). These plots emphasize both the reality of a change in the period distribution at the colors we have advocated in the previous paragraphs and the fact that the location of this effect progresses to redder colors for older clusters.

What is somewhat concerning is that the red branch of the Pleiades slow sequence (the stars with red circles and $\left(V-K_{\mathrm{s}}\right)_{0}>2.9$ in the first panel, or correspondingly the stars with $\Delta P \sim 4$ in the second panel) seems much less populated in M34 and M35. This could be a selection bias-these stars would be precisely the ones most difficult for ground surveys to detect their periodicity because of their expected long periods, relatively small amplitudes, and likely strong spot evolution/migration (as found in the Pleiades - see next few paragraphs). Alternatively, the Pleiades may be unusual for a young cluster in having the red portion of the slow sequence so well populated. In either event,

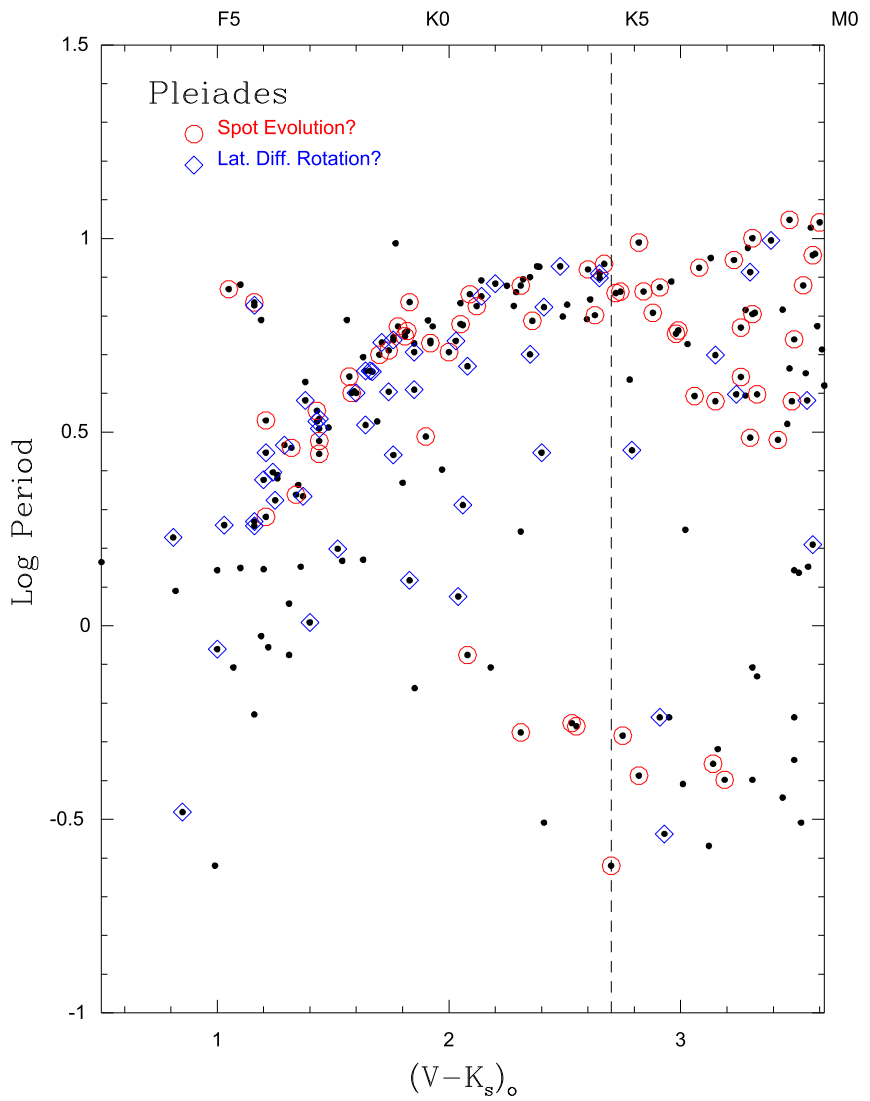

Figure 14. Period-color plot for FGK stars, marking stars with differing lightcurve morphologies as deduced in Papers I and II. Red circles correspond to stars with single-peaked LS periodograms but evolving light-curve shape; blue diamonds correspond to stars with two, close peaks in their LS periodogram (excluding "double-dip" stars). Bluer than $\left(V-K_{\mathrm{s}}\right)_{0}=2.6$, the former signature dominates; redder than that point, the latter signature is significantly more frequent.

this could argue against designating the red edge of the slow sequence $\left(\left(V-K_{\mathrm{s}}\right)_{0}=3.7\right.$ for the Pleiades $)$ as an empirical signpost of age for young clusters. Instead, the color of the kink in the distribution $\left(\left(V-K_{\mathrm{s}}\right)_{0}=2.6,2.9\right.$, and 3.1 for ages of 125, 160 , and $220 \mathrm{Myr}$, respectively) could be a better age indicator. M35 was observed by $K 2$ in Field 0 , and so it may eventually be possible to use those data to derive periods for more late $\mathrm{K}$ dwarfs in that cluster and possibly determine better the shape of its slow sequence and thereby allow a more detailed comparison to the Pleiades rotational data.

If the kink in the Pleiades slow-sequence period-color relation marks a change in magnetic field properties or wind properties, it is reasonable to believe that this change could be reflected in other measureable properties of the $K 2$ light curves. As noted in Section 2 (based on the histograms shown in Figure 3), the fraction of slowly rotating stars does change abruptly at this color, going from $\sim 60 \%$ for $\left(V-K_{\mathrm{S}}\right)_{0}<2.6$ to of order $30 \%$ for $\left(V-K_{\mathrm{s}}\right)_{0}>2.6$. There is also a marked change in the morphology of the $K 2$ light curves at that color. Figure 14 again shows the period versus color plot for the Pleiades, but this time marking stars found in Section 3.4 of Paper II to have two resolved, close peaks in their LS periodogram suggestive of either latitudinal differential rotation or spot evolution (blue diamonds in the figure) and those stars with single-peaked LS periodograms but with evolving light-curve shape (red circles in the figure). A total of $78 \%$ of the FGK stars in the slow, intermediate, and 
rapidly rotating sequences in Figure 2 have one or both of these signatures, versus a negligible fraction for the $\mathrm{M}$ dwarfs. This alone illustrates that the magnetic field morphology differs between the GK dwarfs and the $\mathrm{M}$ dwarfs. Blueward of $\left(V-K_{\mathrm{s}}\right)_{0}=2.6$, most $(54 \%)$ of the symbols are blue, meaning that stars with two close, resolved peaks in their periodograms predominate; redward of $\left(V-K_{\mathrm{s}}\right)_{0}=2.6$, most $(76 \%)$ of the symbols are red, corresponding to single-peaked periodograms and evolving light-curve shape. The driver of the morphology changes in either case could be spot evolution or latitudinal differential rotation or a combination of both-here we only note that there is an empirical change in the frequency of the two morphologies at this $\left(V-K_{\mathrm{s}}\right)_{0} \sim 2$.6. While these effects are not definitive, they hint at a change in the average magnetic field topology at this boundary. Because this boundary appears to move to lower mass with increasing age, it must be driven by some internal process that depends on those two properties.

\section{THE ROTATIONAL PERIODS OF M DWARFS IN THE PLEIADES}

\subsection{Mass-Period Relations for Young M Dwarfs}

Analysis of ground-based rotation period studies of two nearby, young open clusters (NGC 2547, age 40 Myr, Irwin et al. 2008; NGC 2516, age $150 \mathrm{Myr}$, Irwin et al. 2007) has been used to argue that the rotation period distribution for ZAMS-age M dwarfs follows a $J \propto M$ (or, equivalently, a $P \propto M^{2}$ ) relation. Those authors note that stars in this same mass range, $0.1<M / M_{\odot}<0.4$, in star-forming regions with ages of a few megayears also follow a $J \propto M$ relation. The Pleiades $K 2$ data provide a larger sample of well-determined rotation periods in this mass range than are available for any other cluster; we use those data here in order to determine how those periods depend on mass (and how this compares to the stars in NGC 2547 and NGC 2516). We use the Baraffe et al. (2015, hereafter BHAC15) $120 \mathrm{Myr}$ isochrone to estimate masses for the Pleiades stars, as described in Appendix A. We also choose to reexamine how the Pleiades (ZAMS-age) period-mass relation evolves with time by comparing it to period data for NGC 2264 (we describe the NGC 2264 period data and mass determinations in Appendix B). We have compiled our own list of NGC 2264 periods, determined $\left(V-K_{\mathrm{s}}\right)_{0}$ colors for those stars, estimated masses using the same BHAC15 models as for the Pleiades (except now for $3 \mathrm{Myr}$ ), and culled the sample to remove stars with strong IR excesses, large reddening, or that fall below the main locus of NGC 2264 members in a CMD).

Figure 15 shows the rotational velocity distribution of late $\mathrm{M}$ dwarfs in the Pleiades and NGC 2264, in histogram format. Comparison of those diagrams shows that the Pleiades distribution is similar in shape, but somewhat narrower, than the distribution in NGC 2264. That is, the distributions are unimodal (as opposed to the bimodal distribution seen at higher mass), and the 10\%-90\% width of the distribution when plotted in terms of $\log P$ is about 1 dex (but broader for NGC 2264). The Pleiades stars obviously have spun up considerably relative to their younger cousins; the median rotational period at $0.3 M_{\odot}$ in NGC 2264 is about 3.8 days, while it is about 0.68 days in the Pleiades, a change by about a factor of six assuming that the Pleiades and NGC 2264 have the same parent distribution. According to the BHAC15 models, the moment of inertia for a $0.3 M_{\odot}$ star decreases by
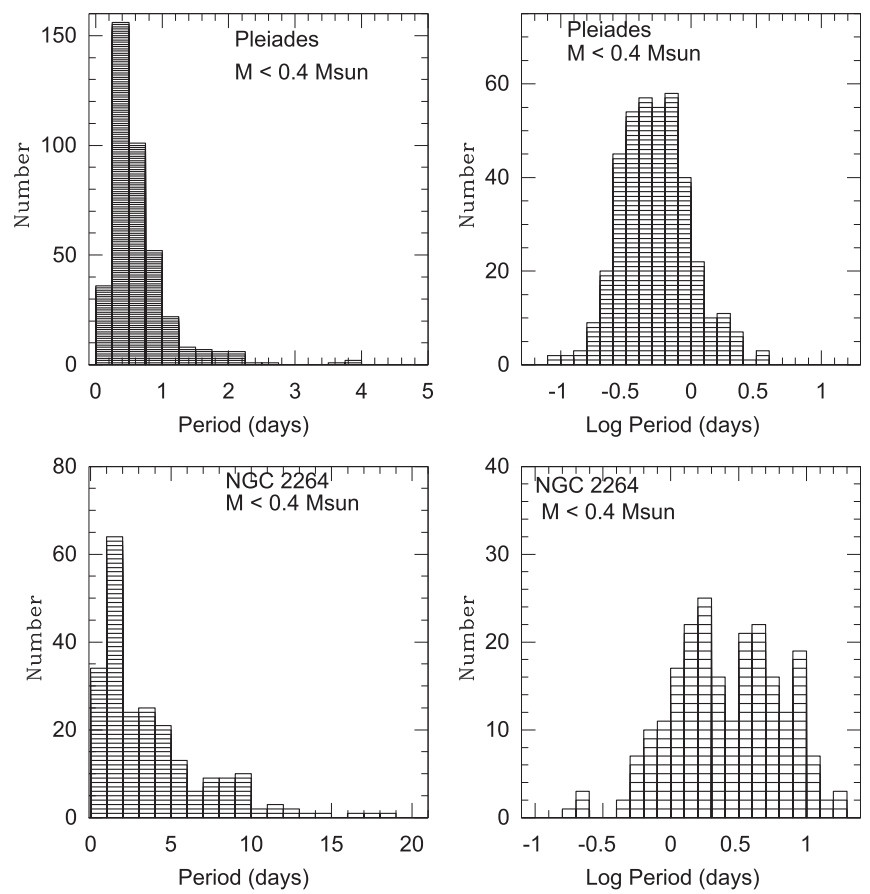

Figure 15. Histograms of the rotation period distribution for late-type $M$ dwarfs in the Pleiades (top) and NGC 2264 (bottom), using both linear (left panels) and logarithmic (right panels) binning. The shape and width of the rotational distribution for late $M$ dwarfs in the Pleiades is similar to that measured for late M dwarfs in the few megayear old NGC 2264, though the distribution in NGC 2264 appears to be somewhat broader.

about a factor of 12 between 3 and 125 Myr. PMS angular momentum loss mechanisms therefore, on average, drain about half of the angular momentum from $0.3 M_{\odot} \mathrm{M}$ dwarfs during this time interval. The similarity in the shape, but somewhat broader distribution in NGC 2264, suggests that angular momentum loss rates scale only weakly with rotation period in this mass and age regime.

In order to determine the dependence of period on mass for the Pleiades $\mathrm{M}$ dwarfs, we have divided the stars into six color bins of 0.4 mag width, beginning at $\left(V-K_{\mathrm{s}}\right)_{0}=4.1$ (approximately $\left.0.6 M_{\odot}\right)$ and ending at $\left(V-K_{\mathrm{s}}\right)_{0}=6.5$ (approximately $\left.0.1 M_{\odot}\right)$, and determined the median period in each color bin. Figure 16 shows these median periods and several possible period-mass power-law relations. For stars somewhat higher in mass than the Sun, Kraft proposed an initial angular momentum distribution of the form $J / M \propto M^{2 / 3}$ (Kraft 1970). With $J \propto M R^{2} \Omega$, and given that at Pleiades age in this mass range it is reasonable to adopt the approximation that $R \propto M$, the Kraft law reduces to $P \propto M^{4 / 3}$. For the Pleiades $M$ dwarfs (Figure 16), the Kraft relation seems a bit too shallow, whereas a $P \propto M^{2}$ relation appears to be too steep. The $P \propto M^{1.5}$ (corresponding to $J \propto M^{1.5}$ ) relation appears to be a reasonably good fit. In Appendix B, Figure 26 shows a similar plot for NGC 2264. In this case, the best fit is for $P \propto M^{1.15}$. Given the uncertainties in estimating masses at young ages, it is not certain that these two results are significantly different. At $3 \mathrm{Myr}, R$ scales about as $M^{0.5}$ (rather than $R \sim M$ on the ZAMS), so our period-mass relation at $3 \mathrm{Myr}$ corresponds approximately to $J \propto M)$. These period and angular momentum power-law relationships are in approximate accord with the previous determinations by Irwin et al. (2008, 2009). 


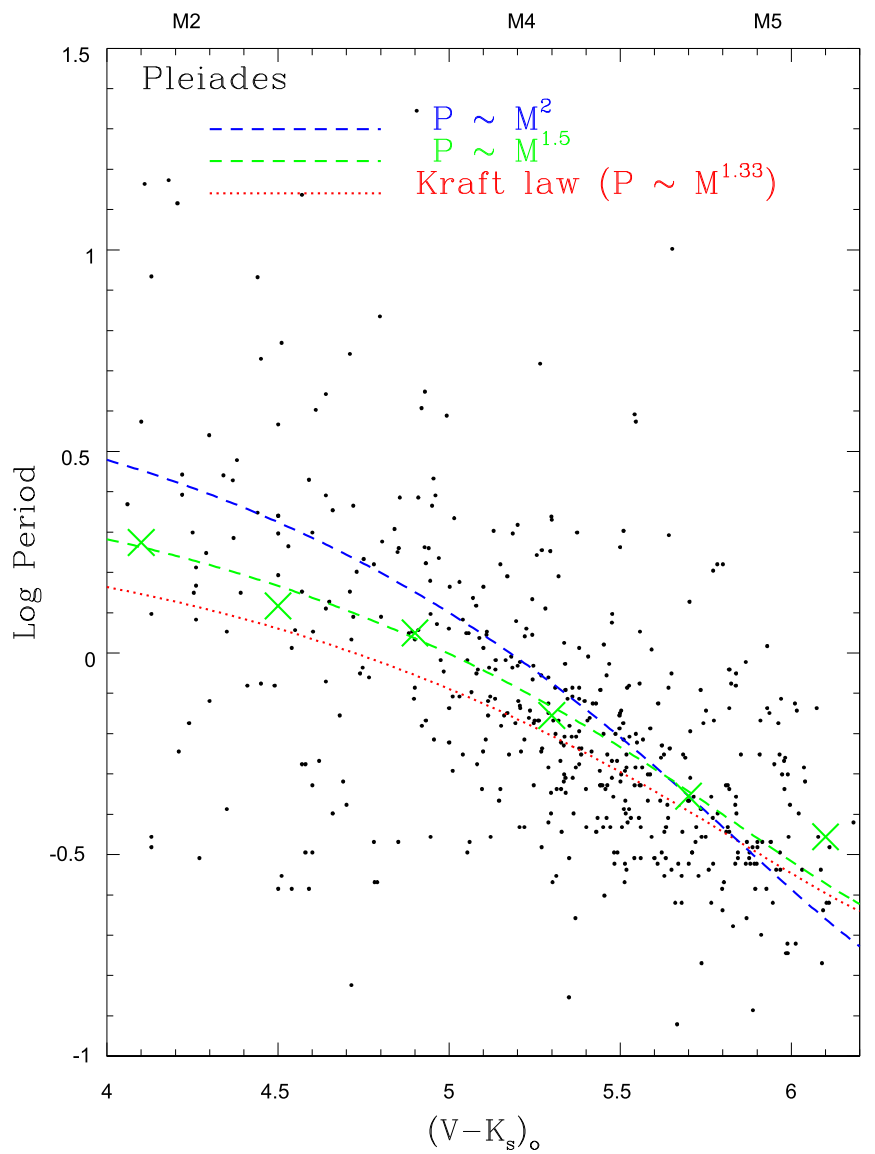

Figure 16. Period-color plot for $M$ dwarfs in the Pleiades. The three curves correspond to three power laws in mass-with exponents of $1.33,1.5$, and 2.0 for red, green, and blue, respectively. The $P \propto M^{1.33}$ relation approximates the Kraft law. The green crosses mark median periods in six color bins of 0.4 mag width beginning at $\left(V-K_{\mathrm{s}}\right)_{0}=4.1$. The mean masses corresponding to each bin are $0.56,0.48,0.40,0.30,0.22$, and $0.17 M_{\odot}$, respectively. The best fit is for $P \propto M^{1.5}$

To illustrate more directly the similarity of the rotational velocity distributions for the $\mathrm{M}$ dwarfs in the two clusters, Figure 17 plots the median periods and best polynomial fits for both NGC 2264 and the Pleiades as a function of mass (as estimated from the BHAC15 isochrones). In this figure, we have divided all the NGC 2264 periods by 5 , in order to approximately account for spin-up between 3 and $125 \mathrm{Myr}$. This scaling succeeds remarkably well in aligning the median periods for the two clusters, indicating that the angular momentum loss mechanism in this mass and age range preserves the functional dependence of rotation period on mass.

The 1 dex width for the late $\mathrm{M}$ rotation distribution in the two clusters is somewhat misleading because much of that width simply reflects the strong correlation between period and mass. The median period at $\left(V-K_{\mathrm{s}}\right)_{0}=5.0$ (median mass $\left.=0.39 M_{\odot}\right)$ is 1.10 days, while at $\left(V-K_{\mathrm{s}}\right)_{0}=5.8$ (median mass $=0.21 M_{\odot}$ ) it is 0.37 days. To get a better measure of the true range in $P$ at a given mass for the late $\mathrm{M}$ dwarfs in the Pleiades, we have subtracted an appropriately normalized power law $\left(P=4.482 \times M^{1.5}\right)$ from the observed periods and zero-point-shifted the periods to the median period at $\left(V-K_{\mathrm{s}}\right)_{0}=5.0$. The logarithmic distribution of these normalized periods is shown in Figure 18, where it can be seen that the width of the distribution is now only about half of

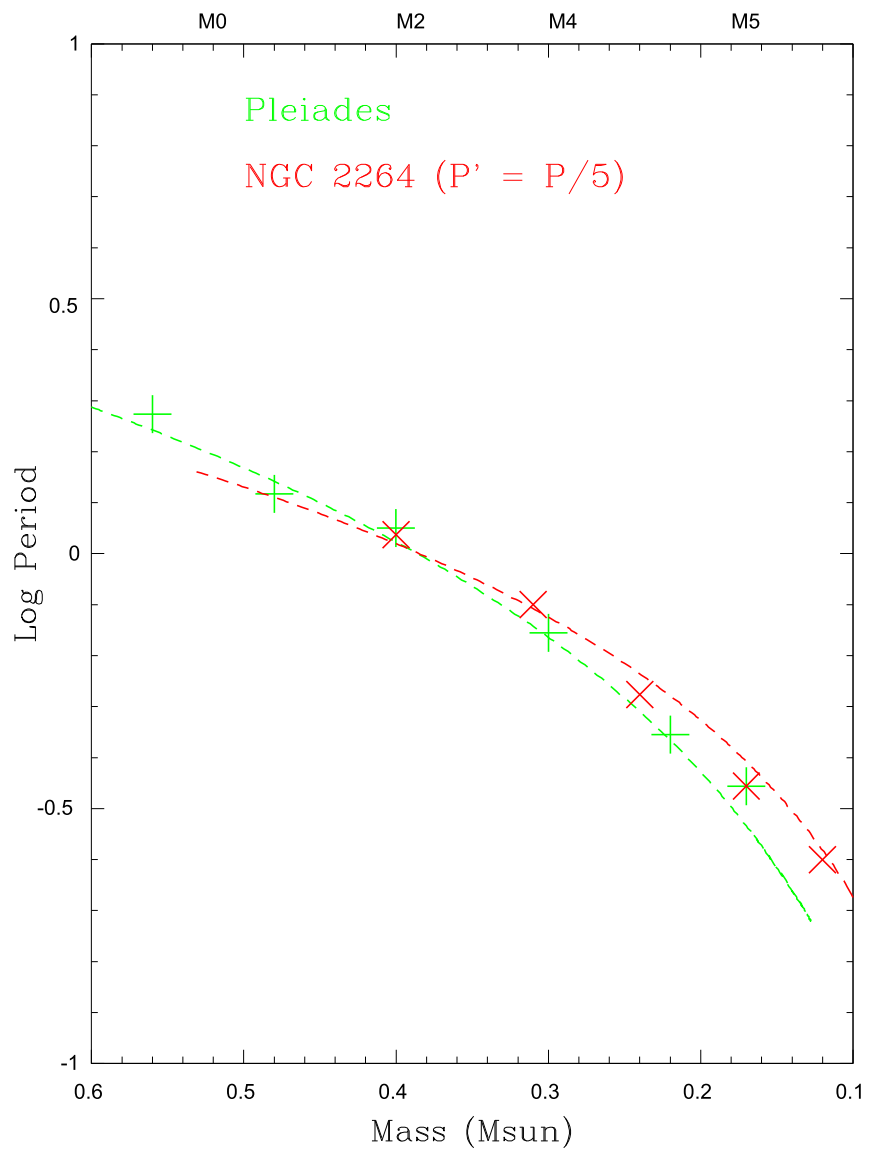

Figure 17. Median periods as a function of mass (as shown in Figure 16 for the Pleiades [green plus signs] and in Figure 26) for NGC 2264 (red crosses), where the masses have been derived from BHAC15 isochrones and the periods for NGC 2264 have been divided by 5 . The two curves are the best polynomial fits shown in Figures 16 and 26, except again dividing the NGC 2264 periods by 5 . When scaled in this fashion, the median periods for the two clusters are very similar.

that deduced without correction for the strong correlation of $P$ with mass.

\subsection{The Initial Angular Momentum Distribution of $M$ Dwarf Binary Stars}

For the Pleiades FGK stars, in Paper II we have associated the presence of close, resolved peaks $(\Delta P / P<0.45)$ in the LS periodogram with latitudinal differential rotation or spot evolution on a single star, and distant, resolved peaks $(\Delta P /$ $P>0.45$ ) with the rotation periods of the two stars in a binary. Theory predicts that fully convective stars should rotate as solid bodies; therefore, we might have simply assumed that all resolved peaks in the LS periodogram for mid- to late $M$ dwarfs were indicative of binary stars. However, we prefer to adopt an empirical approach and allow the data to determine the physical mechanism for multiple peaks in the LS periodogram of late $\mathrm{M}$ dwarfs. We believe that the Pleiades data do require a binary star interpretation, but also that those data have interesting implications for the initial angular momentum distribution of binary $\mathrm{M}$ dwarfs.

For most of the subsequent analysis, we limit ourselves to mid- to late $\mathrm{M}$ dwarfs $\left(5.0<\left(V-K_{\mathrm{s}}\right)_{0}<6.0\right)$, with the blue boundary being set at where one expects stars to become fully convective and the red boundary being set where our period 


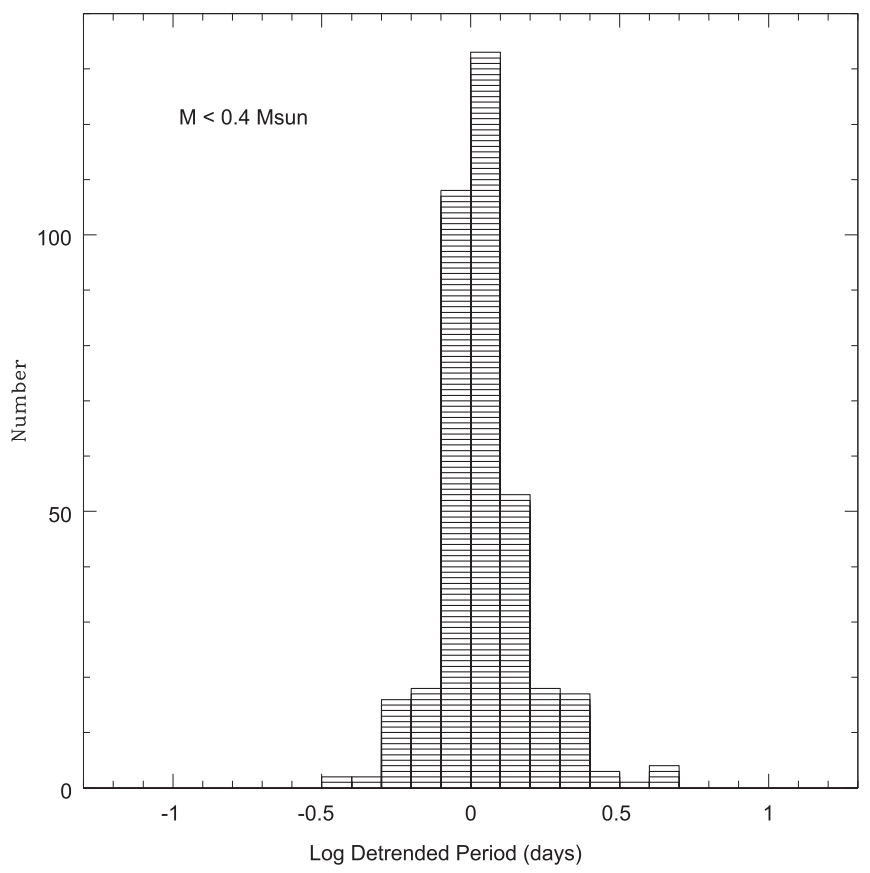

Figure 18. Histogram of periods for stars with $M<0.4 M_{\odot}$ in the Pleiades, after subtracting a power law in mass from the distribution $\left(P=4.482 \times M^{1.5}\right)$, and normalizing to the mean period at $M=0.4 M_{\odot}$. The true (mass independent) width of the distribution is much reduced compared to that shown in Figure 15.

data become significantly incomplete due to the faintness of the stars.

The top left panel of Figure 19 shows where M stars identified in Paper II as having two close peaks in the LS periodogram fall in a period-color diagram. About $10 \%$ of the stars with periods in this color range are identified with this signature. The top right panel of Figure 19 provides the $V$ versus $\left(V-K_{\mathrm{s}}\right)_{0}$ diagram for these same stars, as well as for stars identified in Paper II as having distant resolved peaks in the LS periodogram. Nearly all of the stars in both categories fall well above the single-star locus in the CMD, most easily interpreted as indicating that they are photometric binaries. This diagram alone is prima facie evidence that resolved peaks of any form in this color range should be interpreted as the periods of both components of a binary because we can think of no reason why latitudinal differential rotation should only be detectable on photometric binaries. The only possible escape we can think of for this interpretation would be if the stars with two resolved peaks were more spotted than single-peak stars and if heavily spotted late $\mathrm{M}$ Pleiades members were significantly redder in $\left(V-K_{\mathrm{s}}\right)_{0}$ than otherwise similar Pleiads. The bottom left panel of Figure 19 shows that this escape route is not viable, because the resolved close-peak stars are just as strongly displaced above the single-star locus in a $g$ versus $g-i \mathrm{CMD}$, and one expects $g-i$ to not be significantly affected by spottedness (Stauffer et al. 2003). ${ }^{21}$ Rappaport et al. (2014) also concluded that rapidly rotating, field $M$ dwarfs with two periods detected in their LS

\footnotetext{
21 Spots likely do cause Pleiades $M$ dwarfs, particularly those that are more rapidly rotating, to have redder $\left(V-K_{\mathrm{s}}\right)_{0}$ colors, as emphasized most recently by Covey et al. (2016). However, this effect is too small to explain the quite large CMD displacement for the resolved peaks stars in Figure 19.
}
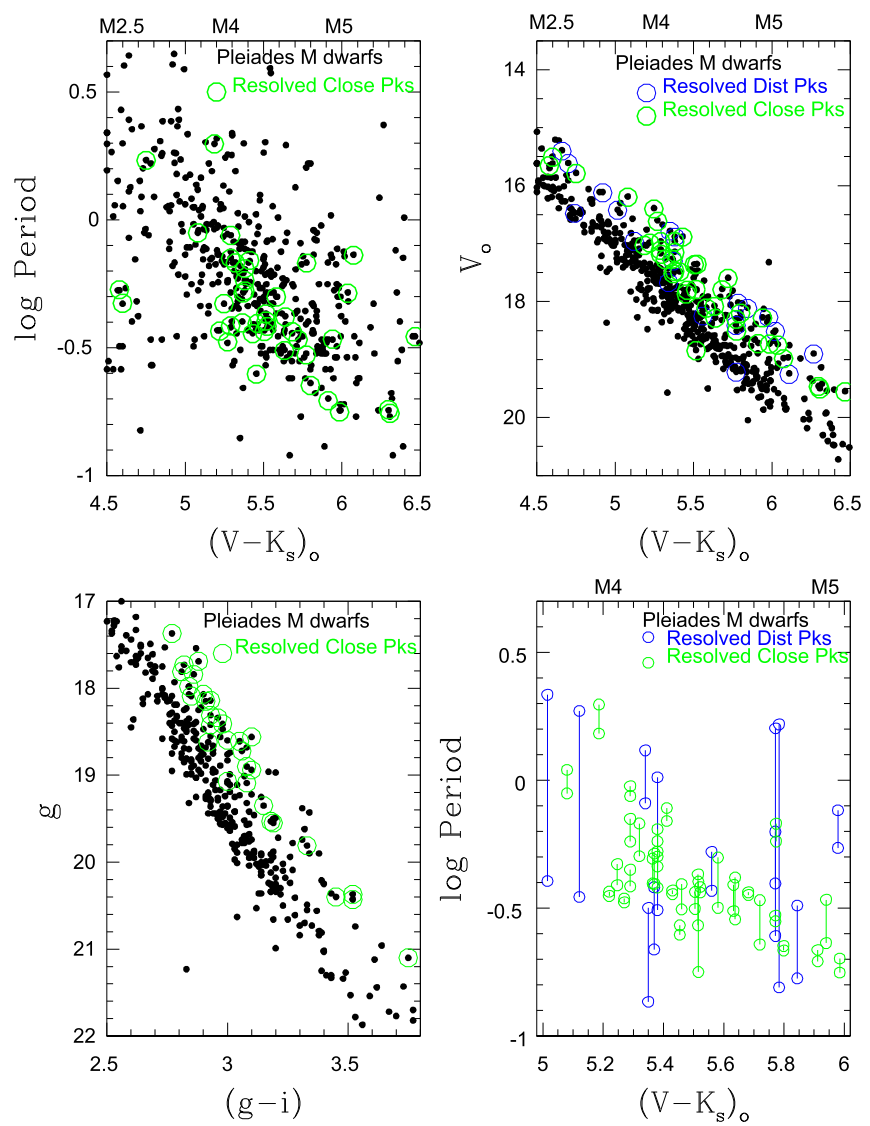

Figure 19. Top left: period-color plot for $M$ dwarfs in the Pleiades, marking stars with two close peaks in their LS periodogram. Note that the stars with two close peaks are, on average, relatively rapid rotators. Top right: CMD for the same set of stars, as well as stars with distant resolved peaks. The vast majority of both groups are displaced well above the single-star sequence, suggesting that they are photometric binaries. Bottom left: a similar CMD, except now for those stars where we have ugri photometry, showing qualitatively similar behavior (and thereby eliminating $\left(V-K_{\mathrm{s}}\right)_{0}$ excesses for the stars with two close peaks as a plausible explanation for their displacements in the second panel). Bottom right: plot showing both periods for each of the relevant stars, which emphasizes that in most cases the two periods are quite close to each other.

periodogram are almost always best interpreted as young binary stars.

There is at least one apparent problem with interpreting all of the resolved peak stars as binaries; this problem is illustrated in the bottom right panel of Figure 19, where we now show only fully convective stars $\left(5.0<\left(V-K_{\mathrm{s}}\right)_{0}<6.0\right)$ and we plot both periods for each star. Comparing the top left and bottom right panels of Figure 19, it seems that the two identified periods for a large fraction of the stars with two resolved LS peaks are too close to each other to have been drawn randomly from the overall distribution. We have run a Monte Carlo simulation to test this assertion, where we created 1000 sets of 45 binaries, with periods chosen randomly from the observed distribution, selecting stars differing in $\left(V-K_{\mathrm{s}}\right)_{0}$ by less than 0.15 . Figure 20 provides two visualizations of the output from this simulation. The top panel of Figure 20 compares the median normalized period difference to the median first period for the simulations and the observed population of two resolved peak Pleiades M dwarfs with $5.0<\left(V-K_{\mathrm{s}}\right)_{0}<6.0$. None of the simulated groups have normalized period differences as small as found for the late $M$ stars with two resolved peaks, 

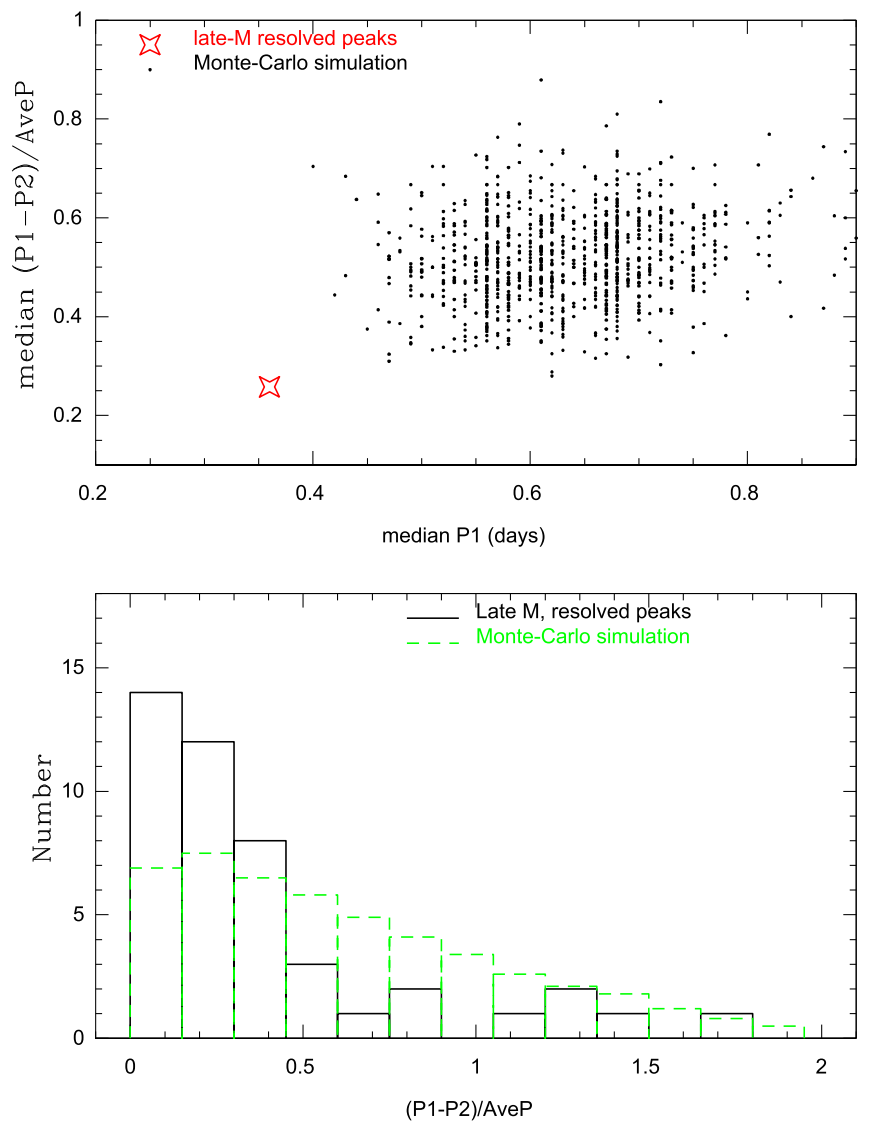

Figure 20. Top: normalized period difference vs. median of the first period for each of the 1000 simulated groups of binaries and for the observed set of late $\mathbf{M}$ Pleiads with two resolved peaks. The Pleiades late $\mathbf{M}$ stars with two resolved peaks have both shorter periods and more closely aligned periods than would have been selected by chance. Bottom: histogram of normalized period difference for the Pleiades late $\mathrm{M}$ dwarfs compared to the average for all the simulation runs, showing a strong excess of small period differences for the Pleiades binaries. Nearly $60 \%$ of the late M Pleiades stars with two resolved peaks in their LS periodogram have period differences less than $30 \%$.

indicating $P<0.1 \%$ for a random selection process. The stars in the resolved peak group also appear to be relatively rapid rotators, with their median period being shorter than the median period of all of the simulation runs. The bottom panel of Figure 20 compares a histogram of the observed normalized period differences to that from the average of the simulated groups. There is a large excess of systems with period differences less than $30 \%$ of the mean period.

A hypothesis that would seem to fit our data is that at the end of the PMS accretion phase, very low mass (VLM) binaries composed of comparable-mass stars have periods that are shorter and more similar to each other than they would be if drawn randomly from all the stars of that mass in their birth group. Because there is little dependence of angular momentum loss rate on either mass or period from that point to the age of the Pleiades (see previous section), this rotation bias is still retained at Pleiades age. At much older ages, where mass- or period-dependent loss rates become important, the similarity of rotation periods may become less apparent-thereby explaining why there has been little evidence for this effect up to now (though from a spectroscopic study of 11 field VLM binary stars, Konopacky et al. [2012] concluded that "the components of VLM binaries are not randomly paired in $v \sin i$ "). The tendency for late $\mathrm{M}$ photometric binary stars to rotate faster than single stars of the same color is in the same sense as we found for $\mathrm{G}$ and $\mathrm{K}$ dwarf binaries in Section 3.1.

\subsection{Inferences for Magnetic Fields and Spot Properties of Pleiades $M$ Dwarfs}

There are suggestive correlations between the distribution of rotation periods in the Pleiades and the measurements of surface magnetic field topologies being made in recent years with ZDI and other techniques (Donati \& Landstreet 2009; Linsky \& Scholler 2015). In particular, those data show a sharp transition in magnetic field topology at about spectral type M4, corresponding to about $\left(V-K_{\mathrm{s}}\right)_{0}=5.0$. Cooler than that boundary, $M$ dwarfs generally have primarily poloidal, axisymmetric fields and less complex small-scale magnetic structures. They also generally show little latitudinal differential rotation. There is also a step up in the large-scale magnetic energy going from early $M$ to mid-M (Morin et al. 2008, 2010). Early M dwarfs have strong toroidal fields and weak poloidal fields, have more small-scale magnetic structure, and often have fairly strong latitudinal differential rotation. They also have more rapid evolution in their magnetic topology, perhaps due to the stronger shearing induced by the differential rotation (Donati et al. 2008). The sharp transition in magnetic properties plausibly occurs at or near the mass where M dwarfs become fully convective $\left(M \sim 0.35 M_{\odot}\right.$; spectral type $\sim \mathrm{M} 3.5$ ).

We have already shown in Figure 14 that the $K 2$ data corroborate that the GK dwarfs very often show signatures of spot evolution or latitudinal differential rotation. There are other morphological features of the $K 2$ light curves that may shed light on the magnetic topologies of Pleiades-age $M$ dwarfs. The top panel of Figure 21 highlights a different facet of light-curve morphology; the stars circled in red in this figure have LS periodograms with just one, narrow peak and phased light curves that show no significant evolution with time and which have sinusoidal or nearly sinusoidal shapes. Light curves of this type are almost absent in the slowly rotating FGK sequence $\left(10 \%\right.$ frequency, for $\left.1.1<\left(V-K_{\mathrm{s}}\right)_{0}<3.7\right)$, whereas among the rapid rotators in the same color range (the blue circled points in Figure 2), 45\% have light curves of this type. Among the early $\mathrm{M}$ dwarfs with $3.7<\left(V-K_{\mathrm{s}}\right)_{0}<4.6,53 \%$ $(48 / 91)$ have this signature, whereas for later $M$ dwarfs with $4.6<\left(V-K_{\mathrm{s}}\right)_{0}<6.0$, the fraction rises to $73 \%(300 / 410)$. The latter number should be regarded as a lower limit because some of the late $\mathrm{M}$ dwarfs are very faint and/or have very low amplitude variability, making it difficult to characterize the morphological status of the light curve. These stable, sinusoidal light curves imply long-lived spot groups at relatively high latitudes.

The bottom panel of Figure 21 highlights a different morphological signature, stars with two or more peaks or minima in their phased light curves and where there is little or no evolution in the light-curve shape over the time period of the full $K 2$ campaign. ${ }^{22}$ That signature is best explained as due to

\footnotetext{
The top panel of Figure 21 is similar to the top left panel of Figure 12 of Paper II. However, the latter figure does not restrict the highlighted stars to being essentially stable over the campaign duration nor to having approximately sinusoidal phased light-curve shapes. Similarly, the bottom panel of Figure 21 is similar to the bottom right panel of Figure 13 in Paper II. Here we show only the stationary "double-dip" stars.
} 

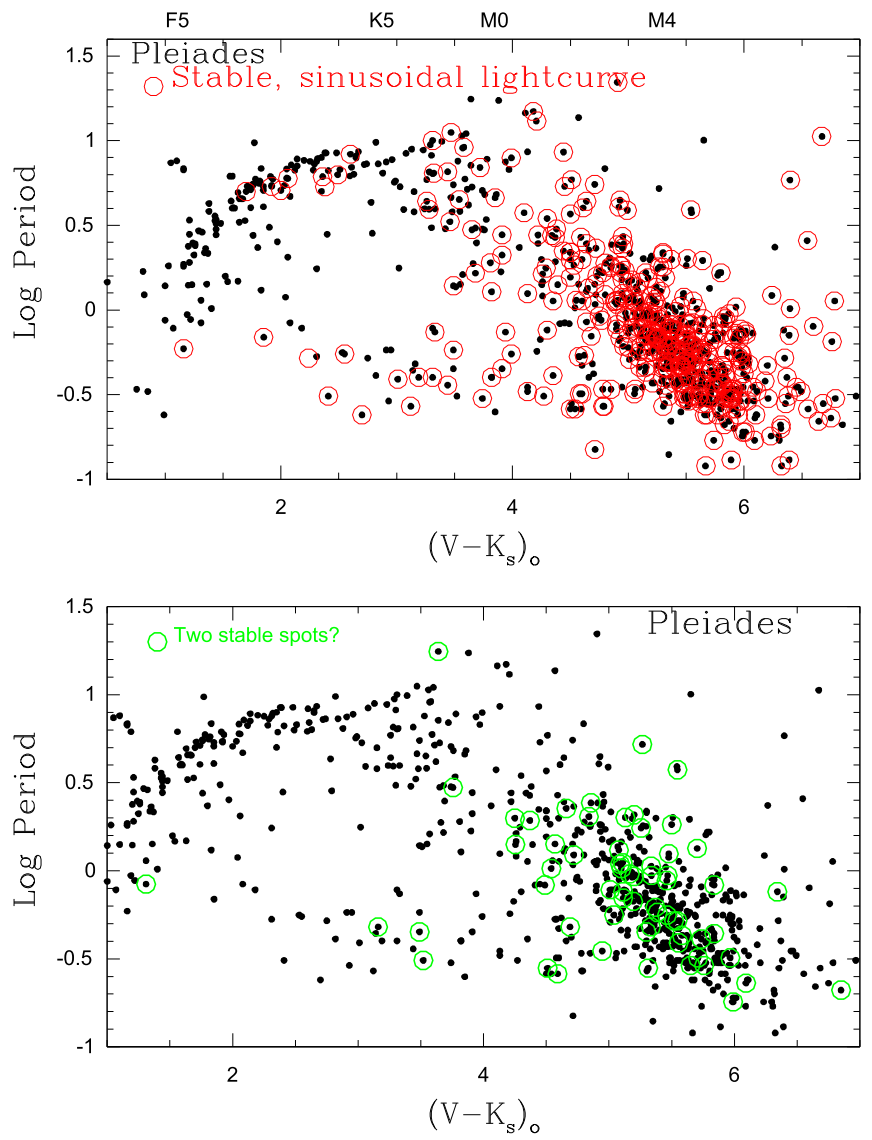

Figure 21. Top: period-color plot for the Pleiades, marking stars with single, narrow peaks in their periodogram and unevolving, normally sinusoidal, light curves. The vast majority of the stars with this signature are $M$ dwarfs. Bottom: period-color plot for the Pleiades, this time marking stars with two peaks or two minima in their phased light curves, best interpreted as having large spot groups in opposite hemispheres of the star. These two light-curve types are the most common morphologies for the rapidly rotating late $\mathrm{M}$ dwarfs at Pleiades age.

the presence of spot groups on opposite hemispheres of the star (see, e.g., Figure 5 of McQuillan et al. 2013 or Figure 4 of Davenport et al. 2015), with little or no differential rotation (and little or no change in spot size or shape).

Our $K 2$ data fit well into the magnetic field topology story line for M dwarfs:

1. There is a striking transition in the period distribution between the early and mid- to late $\mathrm{M}$ dwarfs, at apparently the same place where the B field topologies change. The early $M$ dwarfs show a broad spread in rotation, showing neither a well-defined slow sequence (as present for the FGK dwarfs) nor a well-defined rapidly rotating sequence (as present for the late $\mathrm{M}$ dwarfs).

2. A very large fraction of the late $M$ dwarfs show primarily short periods and stable light curves suggestive of solidbody rotation, long active region lifetimes, and primarily axisymmetric, poloidal fields. Evolving light-curve shapes due to either spot evolution or latitudinal differential rotation are present for about one-quarter of the early $M$ dwarfs (see Figure 13 of Paper II), in agreement with the ZDI measurement of rapid evolution in magnetic topology for some early $\mathrm{M}$ dwarfs.

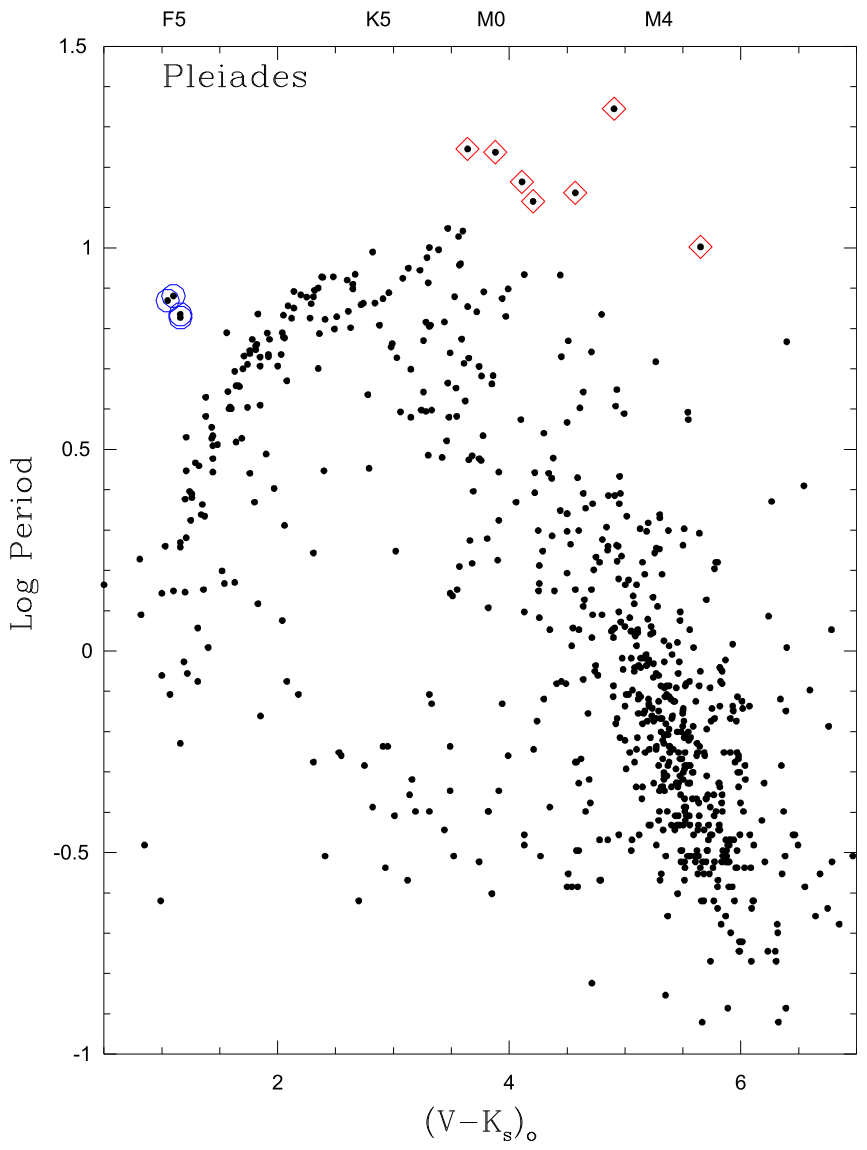

Figure 22. Period-color plot for the Pleiades, highlighting two groups of abnormally slowly rotating stars: blue circles mark a set of very slowly rotating $\mathrm{F}$ dwarfs, and red diamonds mark a set of very slowly rotating $M$ dwarfs. These two sets of stars are discussed in Sections 5.1 and 5.2, respectively.

\section{STARS OF SPECIAL INTEREST}

It is often useful to examine outliers to distributions because their properties may provide clues to not only why they are outliers but also to the physics that sculpts the distribution for the majority of stars. There are two groups of stars whose rotation periods seem abnormally slow compared to the rest of the Pleiades members; these two groups are highlighted in Figure 22. We discuss their properties in the next two sections.

\subsection{Four F Dwarfs with Anomalously Long K2 Periods}

Figure 22 includes four $\mathrm{F}$ dwarfs that seem to be "lost in space"- they all have $\left(V-K_{\mathrm{s}}\right)_{0} \sim 1.1$, spectral type $\sim \mathrm{F} 4$, and periods near 7 days, as opposed to an expected period of order 2 days if they were located on the Pleiades slowly rotating sequence. Table 1 lists the properties of these stars. All four stars appear to be very abnormally slowly rotating. Why is this the case?

We note that the two simplest explanations do not work. First, membership in the Pleiades is not an issue; all four stars are essentially certain members of the cluster. Second, their measured properties as listed in Table 1, and in particular their periods, are well determined.

For two of these stars, HII 1132 and HII 1766, the most probable solution to the conundrum is that the star is a binary, and the $K 2$ period measures the rotation period of the 
Table 1

F Stars with Abnormally Slow Rotation Periods

\begin{tabular}{|c|c|c|c|c|c|c|}
\hline EPIC & Name & Spectral Type & $\begin{array}{c}(B-V)_{o} \\
(\mathrm{mag})\end{array}$ & $\begin{array}{c}\left(V-K_{\mathrm{s}}\right)_{0} \\
(\mathrm{mag})\end{array}$ & $\begin{array}{r}\text { Period } \\
\text { (days) }\end{array}$ & $\begin{array}{c}v \sin i \\
\left(\mathrm{~km} \mathrm{~s}^{-1}\right)\end{array}$ \\
\hline 211121734 & HII 605 & F3 & 0.40 & 1.05 & 7.40 & $\ldots$ \\
\hline 210996505 & HII 1132 & F5 & 0.45 & 1.16 & 6.85 & $>40$ \\
\hline 211072160 & HII 1338 & F3 & 0.42 & 1.10 & 7.60 & $\ldots$ \\
\hline 211138217 & HII 1766 & $\mathrm{~F} 4$ & 0.43 & 1.16 & 6.72 & 22.7 \\
\hline
\end{tabular}

Note. Spectral types from Mendoza (1956), except for HII 1132, which is from Kraft (1967b); photometry and periods from Paper I; $v$ sin $i$ values from Queloz et al. (1998). We have a Keck HIRES spectrum for HII 1132, from which we estimate $v \sin i \sim 50 \mathrm{~km} \mathrm{~s}^{-1}$.

secondary star and not the primary. In fact, for these stars, the measured period cannot be the rotation period for the primary because the $v \sin i$ values for these stars are much larger than their inferred rotational velocity if one adopts the $\sim 7$ day period and a radius of order $1.4 R_{\odot}$ appropriate for their spectral type. Examination of DSS and 2MASS finding charts for all four stars shows no nearby line-of-sight companion that would likely contaminate the $K 2$ photometry.

For HII 1132 and HII 1766, one can perform a thought experiment. If the $K 2$ period derives from a companion star that lies on the slowly rotating FGK sequence polynomial relation, what would its magnitude be in the Kepler band and what amplitude would its variability have to be in order to explain the observed light-curve amplitude (where the $K 2$ data are assumed to include a constant signal from the $\mathrm{F}$ star and the variable light curve from the secondary)? Doing that experiment for HII 1132, we find $\left(V-K_{\mathrm{s}}\right)_{0} \sim 2.3, K_{\mathrm{kep}} \sim 12.0$, and an inferred photometric amplitude of close to $7 \%$ for the secondary. That is a large amplitude for a Pleiades K dwarf, but within the range found for slow rotators (see Figure 12 in Paper I). A similar value would be inferred for HII 1766. This situation only arises in the Pleiades for $\mathrm{F}$ star primaries, where any intrinsic variability of the $\mathrm{F}$ star has very low amplitude (Figure 8) and where plausible, slowly rotating secondary stars a few magnitudes fainter could have relatively large photometric amplitudes. HII 1132 and HII 1766 are both displaced above the single-star $V$ versus $\left(V-I_{\mathrm{c}}\right)_{0}$ main sequence by about $0.3 \mathrm{mag}$, making it possible but not certain that they are binaries with relatively low mass companions.

For HII 1338, instead, we believe there is a different explanation. HII 1338 is a known double-line spectroscopic binary, with a measured orbital period of 7.76 days (Raboud \& Mermilliod 1998). The stars in the system likely have their rotation periods tidally locked (or nearly so) to the orbital period, and that is the physical explanation for their slow rotation rate.

HII 605 is also a known spectroscopic binary (Pearce \& Hill 1975; Liu et al. 1991). The radial velocity amplitude is at least $40 \mathrm{~km} \mathrm{~s}^{-1}$, but no orbital parameters have been measured. It is possible therefore that it also has an orbital period short enough to induce tidal locking at the observed rotation period; alternatively, if the orbital period is long, then the explanation proposed for HII 1132 and HII 1776 may apply.

Finally, we note that HII 1132 has by far the largest IR excess of any known Pleiades member (Rhee et al. 2008). It also has a brown dwarf companion (Rodriguez et al. 2012). While one could invent possible connections between these observables and the very slow rotation rate for HII 1132, with only one object in the sample the connection would be tenuous.

\subsection{The Slowest-rotating M Dwarfs in the Pleiades}

Even in a nearby cluster like the Pleiades, membership studies are imperfect, particularly as one pushes to relatively faint magnitudes. As discussed in Paper I, our original list of potential Pleiades members with $K 2$ light curves included of order 1000 stars. We conducted our own literature search for these stars in order to verify membership, and we ended up eliminating about 150 stars as likely non-members (see Section 2.5 of Paper I). Many of these stars either had no periodic signature in the $K 2$ data or had quite long periods for their spectral type if they were Pleiades members (see Appendix C of Paper I). However, even after that culling, several M dwarfs remain that seem to have rotation periods that are abnormally long; these are the stars marked in red with $P>10.0$ days in Figure 22. Are these stars simply non-members that have slipped past our vetting process, or are they stars that have arrived at Pleiades age with rotation rates that fall outside the norm for their mass?

Table 2 provides basic data for these comparatively slowly rotating $\mathrm{M}$ dwarfs, including membership probabilities from the most recent survey efforts (Lodieu et al. 2012; Bouy et al. 2015) and proper motions from the URAT all sky survey (Zacharias et al. 2015). All of the stars in this table are photometric and proper-motion members of the cluster based on these papers and catalogs, and based on our own photometry. There are essentially no other published data for these stars that provide additional useful information on their cluster membership. To help interpret their periods, we have obtained Keck HIRES spectra for four of these stars: HHJ 353, SK 17, HII 370, and DH 668. The membership of DH 668 is uncertain - our measured radial velocity is slightly discrepant from the mean Pleiades motion, and it has $\mathrm{H} \alpha$ strongly in absorption, more consistent with it being a field star. The other three stars have radial velocities within $\sim 1 \mathrm{~km} \mathrm{~s}^{-1}$ of that expected for Pleiades members. In addition, all three have $\mathrm{H} \alpha$ equivalent widths suggestive of youth (that is, they have either weak $\mathrm{H} \alpha$ emission or "filled-in" $\mathrm{H} \alpha$ ). In Figure 23, we show the $\mathrm{H} \alpha$ profiles for these three stars along with a slowly rotating, late $\mathrm{K}$ dwarf Pleiades member (HII 3030) for which we have a WIYN-Hydra echelle spectrum (published in Terndrup et al. 2000). We do not have a $K 2$ light curve for HII 3030; we include it here because of the resemblance of its $\mathrm{H} \alpha$ profile to that for HII 370 and SK 17 and because its $v \sin i$ indicates that it is a relatively slow rotator. HII 3030, HII 370, and SK 17 all have similar, and slightly unusual, $\mathrm{H} \alpha$ emission profiles consisting of two narrow-emission peaks located in what would have been the wings of their $\mathrm{H} \alpha$ absorption profile if they were not 
Table 2

M Stars with Abnormally Slow Rotation Periods

\begin{tabular}{lcccccccc}
\hline \hline EPIC & $\begin{array}{c}K_{\mathrm{s}} \\
(\mathrm{mag})\end{array}$ & $\begin{array}{c}\text { Period } \\
(\text { days })\end{array}$ & $\begin{array}{c}\left(V-K_{\mathrm{s}}\right)_{0} \\
(\mathrm{mag})\end{array}$ & Bouy & Lodieu & $\mu$ R.A. & $\mu$ Decl. & Name \\
\hline 211074500 & 13.18 & 10.06 & 5.65 & 0.99 & 0.84 & 27 & -48 & BPL 88 \\
210865020 & 11.24 & 13.05 & 4.21 & 0.88 & $\ldots$ & 16 & -43 & UGCS J03411+205117 \\
211007577 & 11.67 & 13.70 & 4.57 & 0.99 & 0.54 & 23 & -49 & HHJ 353 \\
211007344 & 11.08 & 14.58 & 4.11 & 0.98 & 0.72 & 16 & -44 & SK 17 \\
211056297 & 10.45 & 17.28 & 3.88 & 0.99 & $\ldots$ & 10 & -59 & HII 370 \\
210855272 & 10.94 & 17.60 & 3.64 & 0.72 & 0.11 & 11 & -38 & DH 668 \\
210975876 & 12.53 & 22.14 & 4.91 & 0.54 & $\ldots$ & 13 & -41 & UGCS J040550+223553
\end{tabular}

Note. Bouy \& Lodieu columns are membership probabilities from Bouy et al. (2015) and Lodieu et al. (2012), respectively. The columns providing proper motions in R.A. ( $\mu$ R.A.) and decl. ( $\mu$ decl.) are in units of mas $\mathrm{yr}^{-1}$, as provided by the URAT proper-motion survey (Zacharias et al. 2015). The mean cluster motion is $\mu$ R.

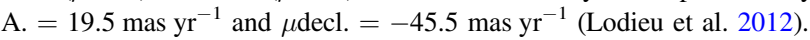
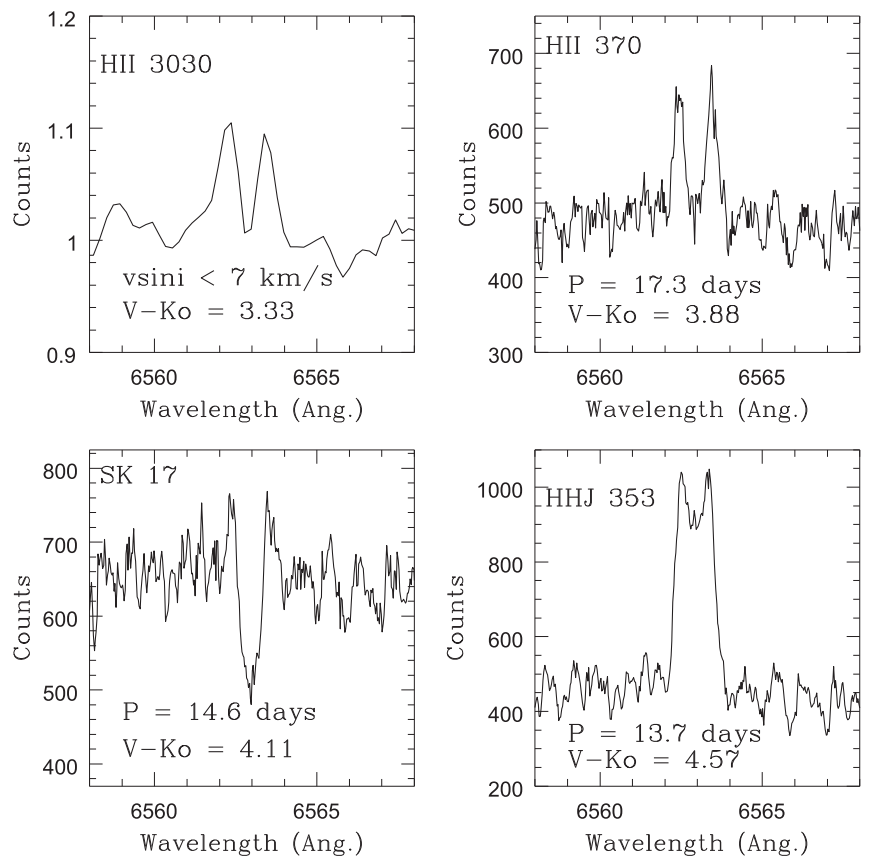

Figure 23. High-resolution spectra centered on $\mathrm{H} \alpha$ for four of the most slowly rotating late $\mathrm{K}$ or early $\mathrm{M}$ dwarfs in the Pleiades. The spectrum for HII 3030, from WIYN/Hydra, is at lower spectral resolution; the other three spectra are from Keck/HIRES.

chromospherically active; HHJ 353 has the strongest $\mathrm{H} \alpha$ emission and has a typical $\mathrm{H} \alpha$ profile for a slowly rotating dMe star. The $\mathrm{H} \alpha$ profiles for HII 3030, HII 370, and SK 17 are essentially as predicted by models for a weakly active dMe star (Cram \& Mullan 1979). The $\mathrm{H} \alpha$ equivalent widths for these four stars are similar to or slightly less than other members for their color. Given that the three stars from Table 2 having these new $\mathrm{H} \alpha$ data are more slowly rotating than any other members of their color, it is probably appropriate that they also have somewhat weaker $\mathrm{H} \alpha$ equivalent widths. We therefore conclude that at least these three-and probably the majority of the others in Table 2are actual Pleiades members, and they are indeed rotating abnormally slowly. Something either in their initial birth environment or in their angular momentum loss between birth and Pleiades age has allowed them to spin down more than all the other stars of their mass.

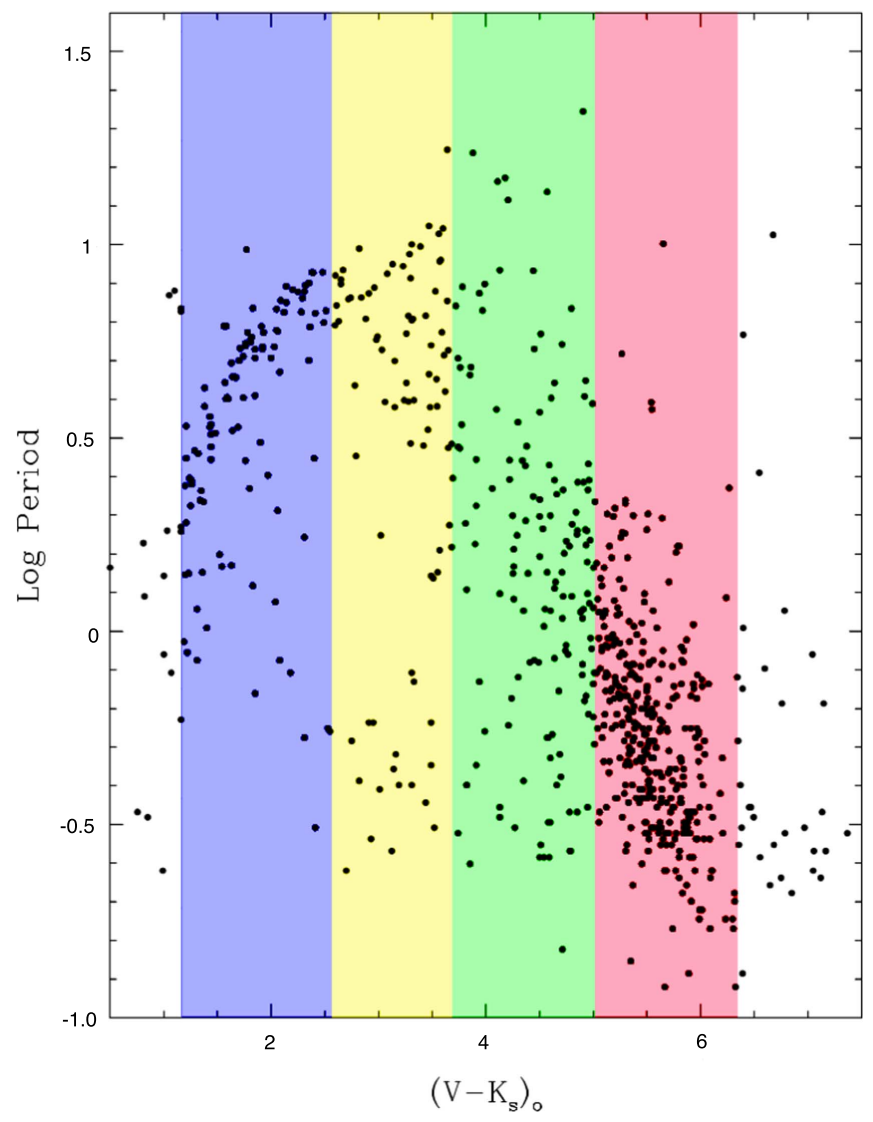

Figure 24. Color coding of the Pleiades period vs. color diagram, highlighting the four mass ranges where stars seem to share similar light-curve morphologies; see text.

\section{SUMMARY AND CONCLUSIONS}

By providing high-cadence, high-precision, long-duration, sensitive, and photometrically stable light curves, the $K 2$ data for the Pleiades have allowed us to derive not only rotation periods and amplitudes but also detailed information from the shape of the light curve not obtainable from ground-based surveys. In order to help frame the discussion of the inferences we have made from the $K 2$ data, we provide in Figure 24 one last rendition of the Pleiades rotation periods, this time using changes in the background color to highlight color (or mass) boundaries where we suspect there are significant changes in 
the magnetic field structure or angular momentum loss rates. As is well known from previous ground-based spectroscopic and photometric surveys, the $\mathrm{G}$ and $\mathrm{K}$ dwarfs in the Pleiades show a bimodal rotation distribution, with large samples of both slowly rotating $(P>2$ days) and rapidly rotating $(P<$ 0.5 days) stars. The $K 2$ data additionally show the following:

1. The slowly rotating sequence in the Pleiades extends across the regions highlighted in blue and yellow in Figure 24, extending from $\left(V-K_{\mathrm{s}}\right)_{0}=1.1$, where $\langle P\rangle=2.0$ days, to $\left(V-K_{\mathrm{s}}\right)_{0}=3.7$, where $\langle P\rangle=11$ days, where the sequence appears to end.

2. Light-curve amplitudes increase sharply redward of $\left(V-K_{\mathrm{s}}\right)_{0}=1.1$ (spectral type F5), which we identify as the color where magnetic dynamos begin to operate efficiently, in agreement with previous conclusions based on spectroscopic activity indicators and X-rays. This is also the color where the Pleiades and Praesepe (age $\sim 600-800$ Myr) slow sequences overlap, indicating that wind-driven angular momentum loss is ineffective blueward of this boundary.

3 . The background color shifts from blue to yellow in Figure 24 to mark a kink in the slowly rotating sequence, where the mean rotation period suddenly decreases by about 1 day. The fraction of stars on the slowly rotating sequence at a given color drops sharply at this boundary, from about $60 \%$ blueward of the boundary to about $30 \%$ redward of the boundary.

4. The much older Praesepe cluster shows a slow sequence displaced throughout the FGK spectral range to slower periods than for the Pleiades and extending much further to the red. Praesepe shows no kink in its slow sequence near $\left(V-K_{\mathrm{s}}\right)_{0}=3$. Plots of the period-color data for the intermediate-age clusters M34 (age 220 Myr) and M35 (age $\sim 160 \mathrm{Myr}$ ) do show possible kinks in their slow sequences at redder colors than in the Pleiades. The location of the kink in the slow sequence may be a better age indicator than the terminal color of that sequence.

5. Most of the stars on the slowly rotating sequence lie close to the single-star locus in $V$ versus $V-I_{\mathrm{C}}$ and $V$ versus $\left(V-K_{\mathrm{s}}\right)_{0}$ CMDs. Many of the stars with rotation periods just slightly shorter than the slow-sequence stars are photometric binaries. One possible explanation for this correlation is that binarity results in shorter disk lifetimes or disks that are otherwise less able to drain angular momentum from their host stars.

6. Most of the stars on the slowly rotating sequence also show evidence of either spot evolution or latitudinal differential rotation in their light curves.

7. The portion of Figure 24 with a red background highlights mid- to late $M$ dwarfs with spectral types roughly M3 to M5. These VLM stars have a unimodal period distribution, with median period decreasing from 1.3 days at $\left(V-K_{\mathrm{s}}\right)_{0}=4.5$ to 0.37 days at $\left(V-K_{\mathrm{s}}\right)_{0}=5.8$. The median $\mathrm{M}$ dwarf rotation periods approximately scale with mass as $P \propto M^{1.5}$, not far from the prediction of the Kraft law proposed to describe the initial angular momentum distribution of high-mass stars.

8. Most $(73 \%)$ of the mid- to late $M$ dwarfs have nearly sinusoidal light-curve shapes that change little or not at all in shape over the duration of the $K 2$ campaign. Such light curves are best interpreted as due to a single spot or spot group located at high latitude. The next most frequent light-curve type in this mass range are stars best interpreted as due to two spots or spot groups, displaced in longitude from each other-but with little evidence for spot evolution or differential rotation.

9. Two close peaks in the LS periodogram are associated with latitudinal differential rotation or spot evolution for FGK stars (see Paper II, Figures 13-15), but are instead best interpreted as the individual periods of components of binary stars among young, late $\mathrm{M}$ dwarfs. We find that the rotation periods of the Pleiades late $\mathrm{M}$ binaries have more similar periods than would be true if their periods were drawn at random from the overall Pleiades $\mathrm{M}$ dwarf distribution.

10. The transition region between where the slow sequence ends and the well-organized, unimodal distribution seen for the mid- to late $\mathrm{M}$ dwarfs begin is marked with a green background in Figure 24. In this transition region (late K to early $\mathrm{M}$ dwarfs), the rotational period distribution is less organized than at any other mass. There is no well-defined slowly rotating sequence, but the distribution still retains some semblance of being bimodal, with relatively slow rotators $(P>1$ day) predominating over rapid rotators $(P<1$ day $)$.

Empirical measurements of the magnetic field structures have begun to illuminate how magnetic geometries change as a function of stellar mass on the main sequence and at very young ages (Donati \& Landstreet 2009; Gregory et al. 2012). However, as yet there are few empirical determinations of the magnetic field structures for low-mass members of the Pleiades or for any other young open cluster (see, however, Folsom et al. 2016, where the first ZDI data on a small sample of Pleiades stars are reported). Some of the light-curve morphological properties we find in the Pleiades roughly match predictions for a solar-type $\alpha-\Omega$ dynamo for the stars in the blue and yellow regions and to some type of distributed dynamo (Chabrier \& Kuker 2006) for the fully convective stars in the red region. The green region then represents a transition regime, where perhaps neither of these two magnetic field generation mechanisms works well.

Our analysis of the Pleiades $K 2$ data emphasizes that while having large samples of well-determined periods is important, it is equally important to also have accurate, homogenous ancillary data in order to eliminate field star contamination and in order to estimate masses (e.g., from broadband colors and extinction corrections) for all of the stars with periods. For the Hyades, Pleiades, Praesepe, and NGC 2516, the reddening is small enough (Stauffer \& Hartmann 1987; Terndrup et al. 2002; Taylor 2006) that whatever uncertainty exists will not significantly affect any conclusions drawn concerning the rotation period distributions. However, for a number of the other clusters with ground-based data, extinctions are much larger and their uncertainty can affect the conclusions one reaches. For example, the extinction to M35 $\left(A_{V}=0.60\right)$ adopted by Meibom et al. (2009) — and accepted in our comparisons and those made by Hartman et al. (2010)_traces back to two unpublished papers; if one were instead to adopt the best published estimate of $A_{V}=0.76$ (Sung \& Bessell 1999), the M35 period-color plot (Figure 13) would suggest an older age. The actual extinction is likely to be in between these two estimates. To make the best use of the marvelous precision in period determinations available from space missions and the best ground campaigns and do precision "gyrochronology" 
Table 3

Derived Data for Pleiades Members with $K 2$ Light Curves

\begin{tabular}{|c|c|c|c|c|c|c|c|c|c|}
\hline Epic & Name & $\begin{array}{c}K_{\mathrm{s}} \\
(\mathrm{mag})\end{array}$ & $\begin{array}{c}\left(V-K_{\mathrm{s}}\right)_{0} \\
(\mathrm{mag})\end{array}$ & $\begin{array}{l}\text { Mass } \\
\left(M_{\odot}\right)\end{array}$ & $M_{\mathrm{bol}}$ & $\begin{array}{c}\text { Radius } \\
\left(R_{\odot}\right)\end{array}$ & $\begin{array}{l}T_{\text {eff }} \\
(\mathrm{K})\end{array}$ & $\begin{array}{c}\Delta V \\
(\mathrm{mag})\end{array}$ & Comments \\
\hline 210754915 & DH 343 & 9.41 & 2.31 & 0.85 & 5.67 & 0.90 & 4911 & 0.36 & \\
\hline 210762863 & DH 318 & 11.04 & 3.94 & 0.59 & 7.80 & 0.60 & 3695 & -0.15 & \\
\hline 210769047 & UGCS J035623.92+192353.3 & 12.88 & 5.71 & 0.22 & 9.80 & 0.35 & 3043 & 0.28 & \\
\hline 210770541 & s4543478 & 11.28 & 5.03 & 0.38 & 8.16 & 0.66 & 3241 & 0.72 & \\
\hline 210776021 & DH 813 & 11.71 & 4.91 & 0.40 & 8.58 & 0.53 & 3284 & 0.16 & \\
\hline 210779549 & PEL S135 & 8.14 & 1.19 & 1.25 & 3.62 & 1.34 & 6454 & 0.30 & \\
\hline 210784223 & s4745026 & 9.97 & 3.12 & 0.73 & 6.53 & 0.84 & 4168 & 0.30 & \\
\hline 210784603 & s3289407 & 10.12 & 3.77 & 0.62 & 6.85 & 0.88 & 3785 & 0.62 & \\
\hline 210791550 & UGCS J035916.64+194427.1 & 12.68 & 5.47 & 0.26 & 9.58 & 0.37 & 3106 & 0.15 & \\
\hline 210803812 & UGCS J033910.21+195530.5 & 12.2 & 5.27 & 0.32 & 9.09 & 0.45 & 3164 & 0.19 & \\
\hline 210804032 & DH 354 & 13.92 & 5.59 & 0.23 & 10.83 & 0.21 & 3073 & -0.84 & \\
\hline
\end{tabular}

(This table is available in its entirety in machine-readable form.)

(Barnes 2010), one also needs to obtain correspondingly good ancillary data.

We view the current paper as but the opening round in a discussion of not just the Pleiades $K 2$ data but more generally the use of high-quality light curves as a means to investigate the physics of angular momentum evolution and magnetic dynamos in young, low-mass stars. We believe that detailed analysis of such light curves for large samples of coeval stars can partner well with ZDI and similar direct measurement of magnetic field topologies to arrive at a better understanding of these topics than either method could do on its own.

A.C.C. acknowledges support from STFC grant ST/ M001296/1. Some of the data presented in this paper were obtained from the Mikulski Archive for Space Telescopes (MAST). Support for MAST for non-HST data is provided by the NASA Office of Space Science via grant NNX09AF08G and by other grants and contracts. This paper includes data collected by the Kepler mission. Funding for the Kepler mission is provided by the NASA Science Mission directorate.

This research has made use of the NASA/IPAC Infrared Science Archive (IRSA), which is operated by the Jet Propulsion Laboratory, California Institute of Technology, under contract with the National Aeronautics and Space Administration. This research has made use of NASA's Astrophysics Data System (ADS) Abstract Service and of the SIMBAD database, operated at CDS, Strasbourg, France. This research has made use of data products from the Two Micron All-Sky Survey (2MASS), which is a joint project of the University of Massachusetts and the Infrared Processing and Analysis Center, funded by the National Aeronautics and Space Administration and the National Science Foundation. The 2MASS data are served by the NASA/IPAC Infrared Science Archive, which is operated by the Jet Propulsion Laboratory, California Institute of Technology, under contract with the National Aeronautics and Space Administration. This publication makes use of data products from the Wide-field Infrared Survey Explorer, which is a joint project of the University of California, Los Angeles, and the Jet Propulsion Laboratory/ California Institute of Technology, funded by the National Aeronautics and Space Administration.

Facilities: K2, Keck, Spitzer.

\section{APPENDIX A MASS ESTIMATES FOR PLEIADES AND NGC 2264 STARS}

We base the mass estimates in both the Pleiades and NGC 2264 on the BHAC15 isochrones, adopting ages of 125 and $3 \mathrm{Myr}$, respectively. We also adopt distances of 136.2 and $760 \mathrm{pc}$ and $A_{v}$ values of 0.12 (Pleiades) and 0.00 (NGC 2264). The latter choice is based, in part, on our culling the member list to exclude stars with significant reddening (see Appendix B).

For both clusters, we use the absolute $K_{\mathrm{s}}$ magnitude as the primary input to the mass estimation. In the Pleiades, we adopt a correction to the $K_{\mathrm{s}}$ magnitude by projecting the observed $K_{\mathrm{s}}$ magnitude down to our adopted single-star $K_{\mathrm{s}}$ versus $\left(V-K_{\mathrm{s}}\right)_{0}$ main-sequence locus illustrated in Figure 4 in order to take binarity into account. This effectively means that our mass estimates for binary stars correspond approximately to the mass of the primary star in the system. We then simply adopt the BHAC15 mass appropriate to that absolute $K_{\mathrm{s}}$ magnitude for $125 \mathrm{Myr}$.

For NGC 2264, we omit the step to correct for binarity simply because there is no well-defined single-star sequence in its CMD.

Table 3 provides masses, radii, bolometric magnitudes, and effective temperatures for the 853 Pleiades members for which we have $K 2$ light curves. The effective temperature estimates are based on the Pecaut \& Mamajek (2013) Table 5 data for main-sequence stars, using $B-V$ to estimate $T_{\text {eff }}$ for the bluest stars and $\left(V-K_{\mathrm{s}}\right)_{0}$ to estimate $T_{\text {eff }}$ for the reddest stars, and a weighted average of the two estimates for $\mathrm{G}$ and $\mathrm{K}$ dwarfs. We adopted the Pecaut \& Mamajek (2013) bolometric corrections $\left(\mathrm{BC}_{V}\right)$ to estimate $M_{\mathrm{Bol}}$, and then the Stefan-Boltzmann law to estimate radii.

\section{APPENDIX B \\ THE ROTATION PERIOD DISTRIBUTION FOR M DWARFS IN NGC 2264}

The Pleiades $\mathrm{M}$ dwarf rotational velocity distribution shows a strong dependence on mass with a relatively small dispersion at fixed mass. Is that distribution primarily a reflection of the initial angular momentum distribution for stars of this mass, or is it a reflection of how angular momentum losses between birth and $125 \mathrm{Myr}$ have shaped the distribution-or do both 

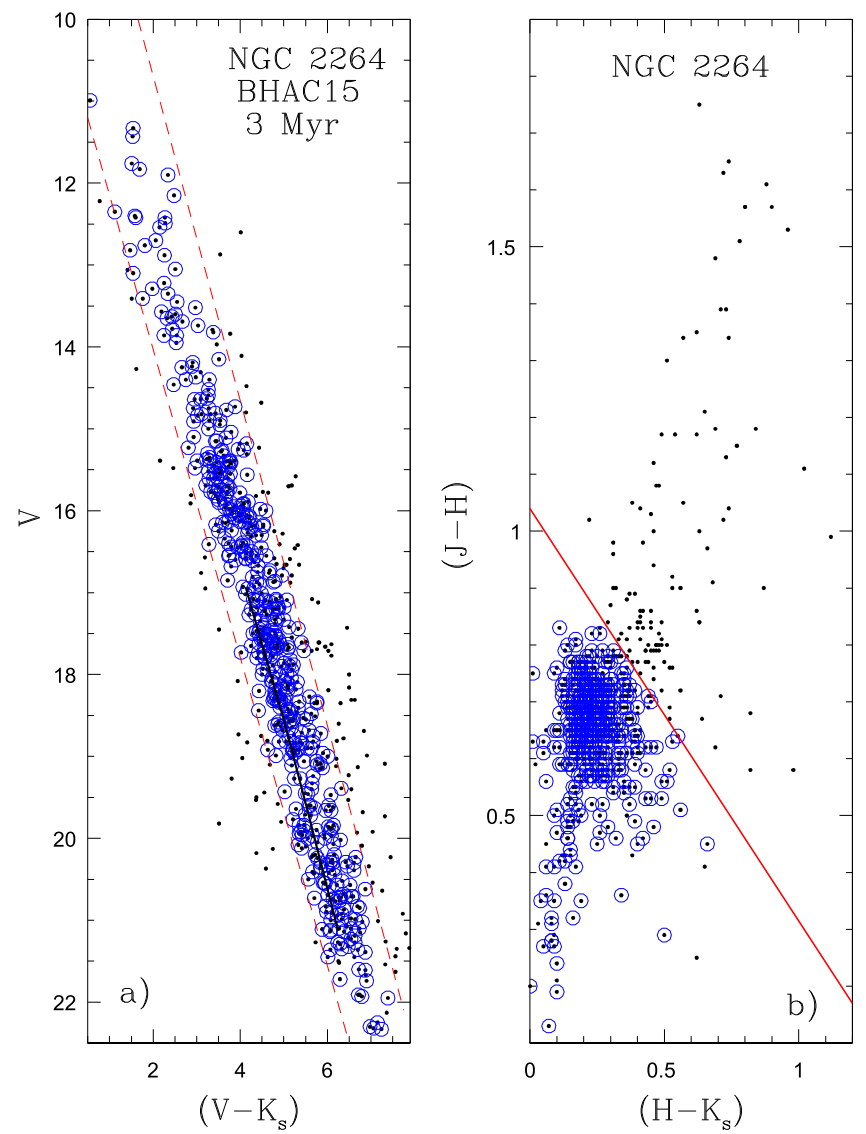

Figure 25. (a) $V$ vs. $\left(V-K_{\mathrm{s}}\right)_{0}$ CMD for the stars for which we have rotation periods in NGC 2264. The two dashed red lines delimit the faint and bright limits within which we retain stars as likely cluster members with an inferred age near 3 Myr. The solid black line is the BHAC15 3 Myr isochrone for 0.5-0.1 $M_{\odot}$. Stars that survive both the optical and the near-IR selection criteria are circled in blue. (b) The near-IR color-color diagram for the same set of stars. Stars above the red line are deleted as either having strong IR exceses or being heavily reddened.

factors contribute significantly to what we see at Pleiades age? In order to partially answer that question, we have gathered together all the relevant rotation data and broadband photometry for one of the best-studied star-forming regionsNGC 2264 - and analyzed those data in essentially the same way as we have treated the Pleiades data. We provide a summary of this process in this appendix; the discussion of the NGC 2264 rotational velocity distribution and its comparison to the Pleiades data appear in Section 4 of the paper.

One difficulty with comparing the Pleiades to NGC 2264 is that no one age can be attached to the stars in NGC 2264; there is instead an obvious spread in ages (see, e.g., Sung \& Bessell 2010), with the youngest being $<1 \mathrm{Myr}$ and the oldest probably being 5 Myr or more. The distance to NGC 2264 is also not as well established as one might think, with even recent published values differing by almost a factor of two. For the present purposes, we simply adopt an age of $3 \mathrm{Myr}$ and a distance of $760 \mathrm{pc}$ (Sung et al. 1997) as values that are representative of the literature.

We compiled rotation periods for members of NGC 2264 from Rebull et al. (2002b), Lamm et al. (2005), and Affer et al. (2013); the first two papers derive their rotation periods from ground-based synoptic photometry, while the third paper used synoptic photometry obtained with the CoRoT satellite. We

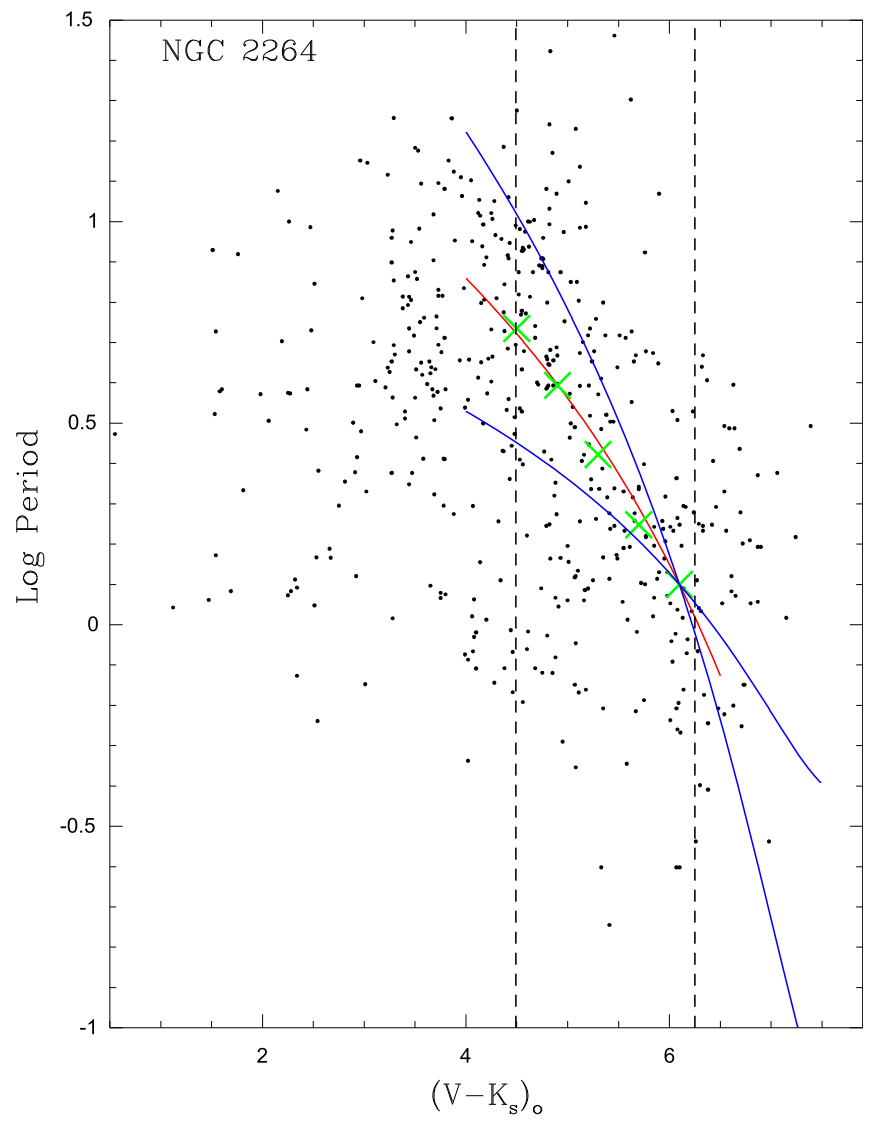

Figure 26. Rotation periods as a function of $\left(V-K_{\mathrm{s}}\right)_{0}$ for NGC 2264, for stars selected to have small reddening, to lack strong IR excesses, and to have colors and magnitudes consistent with the BHAC15 3 Myr isochrone. The vertical dashed lines mark the color corresponding to 0.4 and $0.1 M_{\odot}$; the green crosses are the median periods for all stars in 0.4 mag color bins beginning at $\left(V-K_{\mathrm{s}}\right)_{0}=4.3-4.7$; the mean masses for each color bin are 0.40, 0.31, 0.24, 0.17 , and $0.12 M_{\odot}$, respectively. The red curve corresponds to a $P \propto M^{1.15}$ power law and is the best fit. The other two curves are power laws in mass for exponents of 0.65 and 1.65 .

adopted broadband optical photometry for those stars from Rebull et al. (2002a), and from Sung et al. (2008) we merged those data with $J H K_{\mathrm{s}}$ photometry from the 2MASS pointsource catalog.

Because some of the NGC 2264 stars are classical T Tauri stars and have IR excesses, and some of the NGC 2264 stars are significantly reddened due to their being embedded within or behind molecular clouds, their colors do not necessarily provide an accurate measure of their photospheric temperature (and hence of their inferred mass). Some NGC 2264 members may be much younger or older than average, and it would be preferable to remove those that are most discrepant (this step also would preferentially remove field stars that have been mistaken as members). We therefore first cull the sample of stars to remove outliers, using an optical CMD and the $J-H$ versus $H-K_{s}$ color-color diagram. These two figures are shown in Figure 25, where we illustrate the cuts in color and magnitude we have made to purify the sample of stars from which to determine the $3 \mathrm{Myr}$ rotational velocity distribution.

The period-color diagram for the stars that survived our culling steps (the stars that are not very young, not very old, not very reddened, and lacking strong IR excesses) is shown in Figure 26. The vertical dashed lines mark the color 
Table 4

HIRES Spectral Data for Slowly Rotating Candidate Pleiades Members

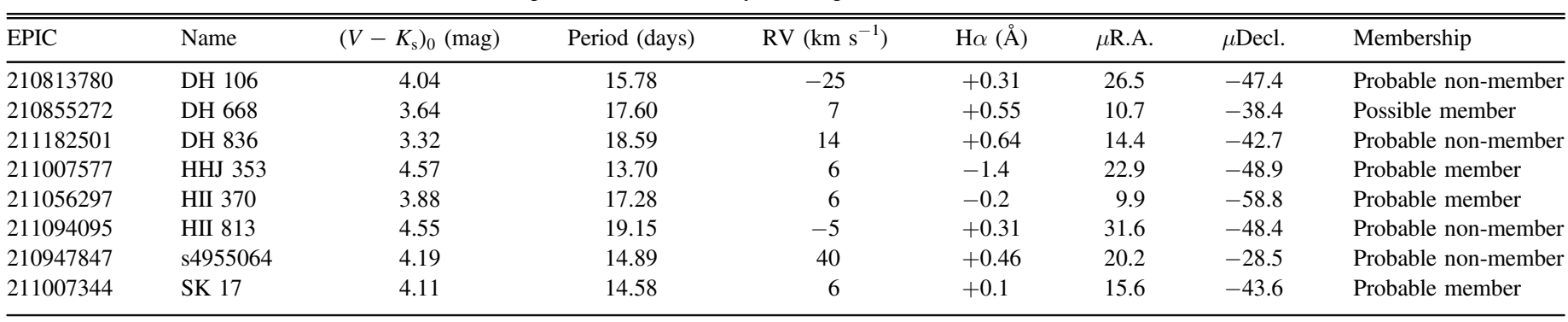

Note. Columns providing proper motions in R.A. ( $\mu$ R.A.) and decl. ( $\mu$ decl.) are in units of mas $\mathrm{yr}^{-1}$, as provided by the URAT proper-motion survey (Zacharias

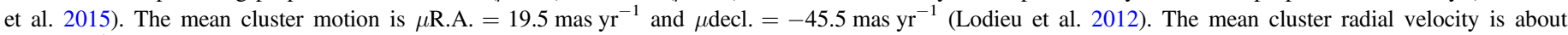
$5.5 \mathrm{~km} \mathrm{~s}^{-1}$.

corresponding to 0.4 and $0.1 M_{\odot}$ according to the BHAC15 $3 \mathrm{Myr}$ isochrone. We have divided the sample into color bins of 0.4 mag width (e.g., $\left(V-K_{\mathrm{s}}\right)_{0}=4.3-4.7,4.7-5.1,5.1-5.5$, etc.), and determined the median rotation period in each bin; those medians are shown as green crosses in Figure 26. Also shown in the figure are curves for three power-law relations between period and mass $-P \propto M, P \propto M^{1.15}$, and $P \propto M^{1.5}$ - all forced to pass through the median period for the reddest color bin in NGC 2264. The $P \propto M^{1.15}$ relation fits the data best.

Finally, we have also constructed histograms of the period distribution for the $0.1-0.4 M_{\odot}$ NGC 2264 members, in order to directly compare to the similar diagrams created from our Pleiades $K 2$ data. These diagrams are presented in Section 4.

\section{APPENDIX C \\ NEW SPECTRA FOR VERY SLOWLY ROTATING CANDIDATE MEMBERS OF THE PLEIADES}

In our initial plots of the $K 2$ periods for Pleiades candidate members, there were about a dozen early $\mathbf{M}$ dwarfs with seemingly unusually long periods. In general, these were stars with very little literature information and whose membership in the Pleiades was therefore uncertain. We obtained highdispersion spectra for eight of these stars with Keck I and HIRES (Vogt et al. 1994) on 2016 February 2-3 in order to use those spectra to help ascertain membership status. The instrument was configured to produce spectra from $\sim 4800$ to $9200 \AA$ using the C5 decker, which provides spectral resolution of $\sim 36,000$. We used FXCOR within IRAF to measure radial velocities, and SPLOT within IRAF to measure the equivalent widths for $\mathrm{H} \alpha$ and to also examine the $\mathrm{H} \alpha$ profile. We also examined the spectra to check whether the Li I $\lambda 6708$ doublet was detected; none of the stars showed Li, which was as expected given their spectral types. Table 4 provides a summary of the measurements we made for these stars and our conclusions concerning membership. We find that three of the stars appear to be good Pleiades members, four are most likely non-members, and one is a possible member. $\mathrm{H} \alpha$ profiles for the three probable members are shown in Figure 22.

\section{REFERENCES}

Affer, L., Micela, G., Favata, F., et al. 2013, MNRAS, 430, 1433 Agueros, M., Covey, K., Lemonias, J., et al. 2011, ApJ, 740, 110 Aguirre, V., Casagrande, L., \& Miglio, A. 2014, IAUS, 298, 375 Anderson, C., Stoeckly, R., \& Kraft, R. 1966, ApJ, 143, 299
Balona, L., Daszynska-Daszkiewicz, J., \& Pamyatnykh, A. 2015, MNRAS, 452, 3073

Balona, L., Guzik, J., Uytterhoeven, K., et al. 2011, MNRAS, 415, 3531

Baraffe, I., Homeier, D., Allard, F., \& Chabrier, G. 2015, A\&A, 577, 42 (BHAC15).

Barnes, S. 2010, ApJ, 722, 222

Barnes, S., Weingrill, J., Granzer, T., et al. 2015, A\&A, 583, 73

Batalha, N. 2014, PNAS, 111, 12647

Bouvier, J., Alencar, S., Harries, T., et al. 2007, in Protostars and Planets V, ed. B. Reipurth, D. Jewitt, \& K. Keil (Tucson, AZ: Univ. Arizona Press), 479 Bouvier, J., Forestini, M., \& Allain, S. 1997, A\&A, 326, 1023

Bouvier, J., Matt, S., Mohanty, S., et al. 2014, in Protostars and Planets VI, ed. H. Beuther, et al. (Tucson, AZ: Univ. of Arizona Press), 433

Bouy, H., Bertin, E., Sarro, L., et al. 2015, A\&A, 577, 148

Brandt, T., \& Huang, C. 2015, ApJ, 807, 24

Carpenter, J. M., Hillenbrand, L. A., \& Skrutskie, M. F. 2001, AJ, 121, 3160 Chabrier, G., \& Kuker, M. 2006, A\&A, 446, 1027

Collier Cameron, A., Campbell, C., \& Quaintrell, H. 1995, A\&A, 298, 133

Collier Cameron, A., \& Jianke, L. 1994, MNRAS, 269, 1099

Covey, K., Agueros, M., Law, N., et al. 2016, ApJ, 822, 81

Cram, L., \& Mullan, D. 1979, ApJ, 234, 579

Davenport, J., Hebb, L., \& Hawley, S. 2015, ApJ, 806, 212

Delorme, P., Collier-Cameron, A., Hebb, L., et al. 2011, MNRAS, 412, 2218

Donati, J.-F., \& Landstreet, J. 2009, ARA\&A, 47, 333

Donati, J.-F., Morin, J., Petit, P., et al. 2008, MNRAS, 390, 545

Douglas, S., Agueros, M., Covey, K., et al. 2016, ApJ, 822, 47

Dupret, M., Grigahcene, A., Garrido, R., et al. 2004, A\&A, 414, L17

Dziembowski, W. 1977, AcA, 27, 95

Folsom, C., Petit, P., Bouvier, J., et al. 2016, arXiv:1601.00684

Gallet, F., \& Bouvier, J. 2013, A\&A, 556, 36

Gallet, F., \& Bouvier, J. 2015, A\&A, 577, 98

Ghosh, P., \& Lamb, F. 1979, ApJ, 234, 296

Gizis, J., Dettman, K., Burgasser, A., et al. 2015, ApJ, 813, 104

Gregory, S., Donati, J., Morin, J., et al. 2012, ApJ, 755, 97

Hartman, J., Bakos, G., Kovacs, G., \& Noyes, R. 2010, MNRAS, 408, 475

Hartman, J., Gaudi, S., Pinsonneault, M., et al. 2009, ApJ, 691, 342

Herbst, W., Bailer-Jones, C., \& Mundt, R. 2001, ApJL, 554, L197

Herbst, W., Eisloffel, J., Mundt, R., \& Scholz, A. 2007, Protostars and Planets V

Howell, S., Sobeck, C., Haas, M., et al. 2014, PASP, 126, 398

Irwin, J., Aigrain, S., Bouvier, J., et al. 2009, MNRAS, 392, 1456

Irwin, J., Hodgkin, S., Aigrain, S., et al. 2007, MNRAS, 377, 741

Irwin, J., Hodgkin, S., Aigrain, S., et al. 2008, MNRAS, 383, 1588

Johnstone, C., Gudel, M., Brott, I., et al. 2015, A\&A, 577, 28

Kamai, B., Vrba, F., Stauffer, J., et al. 2014, AJ, 148, 30

Kaye, A., Handler, G., Krisciunas, K., et al. 1999, PASP, 111, 840

Königl, A. 1991, ApJL, 370, L39

Konopacky, Q., Ghez, A., Fabrycky, D., et al. 2012, ApJ, 750, 79

Kraft, R. 1965, ApJ, 142, 681

Kraft, R. 1966, AJ, 71, 861

Kraft, R. 1967a, ApJ, 148, 129

Kraft, R. 1967b, ApJ, 150, 551

Kraft, R. 1970, in Spectroscopic Astrophysics, an Assessment of the Contributions of Otto Struve, ed. G. Herbig (Berkeley, CA: Univ. California), 385

Lamm, M., Mundt, R., Bailer-Jones, \& Herbst, W. 2005, A\&A, 430, 1005

Linsky, J., \& Scholler, M. 2015, SSRv, 191, 27 
Liu, T., Janes, K., \& Bania, T. 1991, ApJ, 377, 141

Lodieu, N., Deacon, N., Hambly, N., et al. 2012, MNRAS, 422, 1495

Maehara, H., Shibayama, T., Notsu, S., et al. 2012, Natur, 485, 478

McQuillan, A., Aigrain, S., \& Mazeh, T. 2013, MNRAS, 432, 1203

McQuillan, A., Mazeh, T., \& Aigrain, S. 2014, ApJS, 211, 24

Meibom, S., Grundahl, F., Clausen, C., et al. 2009, AJ, 137, 5086

Meibom, S., Mathieu, R., \& Stassun, K. 2007, ApJL, 665, L155

Meibom, S., Mathieu, R., Stassun, K., et al. 2011, ApJ, 733, 115

Melis, C., Reid, M., Mioduszewski, A., et al. 2014, Sci, 345, 1029

Mendoza, E. 1956, ApJ, 123, 54

Messina, S., Parihar, P., Koo, J., et al. 2010, A\&A, 513, 29

Morin, J., Donati, J.-F., Petit, P., et al. 2008, MNRAS, 390, 567

Morin, J., Donati, J.-F., Petit, P., et al. 2010, MNRAS, 407, 2269

Mould, J. 1976, A\&A, 48, 443

Patience, J., Ghez, A., Reid, I., et al. 2002, AJ, 123, 1570

Pearce, J., \& Hill, G. 1975, PDAO, 14, 319

Pecaut, M., \& Mamajek, E. 2013, ApJS, 208, 9

Pei, L., Barth, A., Aldering, G., et al. 2014, ApJ, 795, 38

Prsa, A., Batalha, N., Slawson, R., et al. 2011, AJ, 141, 83

Pye, J., Hodgkin, S., Stern, R., et al. 1994, MNRAS, 266, 798

Queloz, D., Allain, S., Mermilliod, J., et al. 1998, A\&A, 335, 183

Raboud, D., \& Mermilliod, J. 1998, A\&A, 329, 101

Radick, R., Thompson, D., Lockwood, G., et al. 1987, ApJ, 321, 459

Rappaport, S., Swift, J., Levine, A., et al. 2014, ApJ, 788, 114

Rebull, L., Makidon, R., Strom, S., et al. 2002a, AJ, 123, 1528

Rebull, L., Stauffer, J., Bouvier, J., et al. 2016a, AJ, 152, 113 (Paper I)

Rebull, L., Stauffer, J., Bouvier, J., et al. 2016b, AJ, 152, 114 (Paper II)

Rebull, L., Wolff, S., Strom, S., \& Makidon, R. 2002b, AJ, 124, 546
Reinhold, T., Reiners, A., \& Basri, G. 2013, A\&A, 560, A4

Rhee, J., Song, I., \& Zuckerman, B. 2008, ApJ, 675, 777

Rodriguez, D., Marois, C., Zuckerman, B., et al. 2012, ApJ, 748, 30

Rodriguez-Ledesma, M., Mundt, R., \& Eisloffel, J. 2009, A\&A, 502, 883

Rowe, J., Coughlin, J., Victoria, A., et al. 2015, ApJS, 217, 16

Sarro, L., Bouy, H., Berihuete, A., et al. 2014, A\&A, 563, 45

Schatzmann, E. 1962, AnAp, 28, 18

Sierchio, J., Rieke, G., Su, K., et al. 2010, ApJ, 712, 1421

Sills, A., Pinsonneault, M., \& Terndrup, D. 2000, ApJ, 534, 335

Simon, A., Rowe, J., Gaulme, P., et al. 2016, ApJ, 817, 162

Skumanich, A. 1972, ApJ, 171, 565

Soderblom, D., Stauffer, J., Hudon, J., \& Jones, B. 1993a, ApJS, 85, 315

Soderblom, D., Stauffer, J., Macgregor, K., \& Jones, B. 1993b, ApJ, 409, 624

Stauffer, J., \& Hartmann, L. 1987, ApJ, 318, 337

Stauffer, J., Hartmann, L., \& Latham, D. 1987, ApJL, 320, L51

Stauffer, J., Jones, B., Backman, D., et al. 2003, AJ, 126, 833

Stauffer, J., Schulz, G., \& Kirkpatrick, J. D. 1998, ApJL, 499, L199

Sung, H., \& Bessell, M. 1999, MNRAS, 306, 361

Sung, H., Bessell, M., Chun, M., et al. 2008, AJ, 135, 441

Sung, H., Bessell, M., \& Lee, S.-W. 1997, AJ, 114, 2644

Taylor, B. 2006, AJ, 132, 2453

Terndrup, D., Pinsonneault, M., Jeffries, R., et al. 2002, ApJ, 576, 950

Terndrup, D., Stauffer, J., Pinsonneault, M., et al. 2000, AJ, 119, 1303

Vogt, S., Allen, S., Bigelow, B., et al. 1994, Proc SPIE, 2698, 362

Weber, E., \& Davis, L. 1967, ApJ, 148, 217

Wilson, O. C. 1966, ApJ, 144, 695

Zacharias, N., Finch, C., Subasavage, J., et al. 2015, AJ, 150, 101 